\title{
Company Overview for 2017-2021: Apple vs Microsoft
}

\author{
Munisa Abdukarimova ${ }^{1}$, Prof. Abduraim Vahobov ${ }^{2}$, Prof. Vijay Shenai ${ }^{3}$ \\ ${ }^{1}$ Samarkand Institute of Economics and Service \\ ${ }^{2}$ National University of Uzbekistan \\ ${ }^{3}$ Cardiff Metropolitan University
}

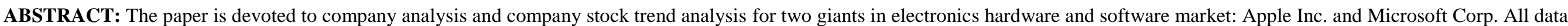

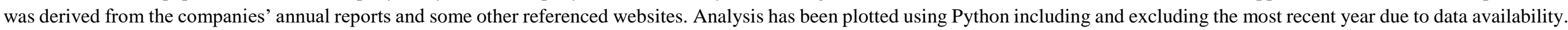

KEYWORDS: Apple Inc., Microsoft Corp., Financial Analysis

\section{SALES AND STOCK MARKET DEVELOPMENT}

In 1976 Steve Jobs, Steve Wozniak and Ronald Wayne founded Apple Computer Inc., renamed as Apple Inc. in 2007, which corporate headquarters located in Cupertino, California. The company started its business initially with personal computers and laser printers, from 2001 presented its portable digital audio players, iPods, iTouchs, iPhones and iPads and in 2011, services such as Siri and iCloud. Apple Inc. has been significantly pioneering in highly competitive electronics market through more dedicated and loyal customer base and continually innovating its products. It also significantly improved it software products such as AirPlay, FaceTime, iCloud, iCloud Drive, iOS, iPadOS, macOS, Xcode.

Currently Apple's assigned CEO is Tim Cook. As for FY2020 it has around 147,000 employees, operates its manufacturing mainly in China and manages its sales through more than 450 retail stores in 16 countries besides its third-party cellular network carriers, wholesalers, and online distribution markets.

The iPhone represents over 50\% of Apple's revenue in 2020, though iPhone sales recently slumped, dropping from $\$ 142$ billion in 2019 to $\$ 137$ billion in 2020 . Apple charges premium prices across all its products, which cause lower-priced smartphones take market share in developing countries. Apple has also long been criticized for its incompatibility with other software.

Despite Apple stores closed in China as a result of the coronavirus, total sales for 2020 has not been plummeted from total sales in 2019. 
International Journal of Current Science Research and Review

ISSN: 2581-8341

Volume 04 Issue 07 July 2021

DOI: 10.47191/ijcsrr/V4-i7-21, Impact Factor: 5.825

IJCSRR @ 2021

www.ijcsrr.org

Table 1. Apple sales by products through 2017-2020 years, in thousand dollars (Adapted from the annual reports of Apple Inc.)

\begin{tabular}{|c|c|c|c|c|c|}
\hline year & iphone & $\operatorname{mac}$ & ipad & wearables & services \\
\hline 2020 & 137781 & 28622 & 23724 & 30620 & 53768 \\
\hline 2019 & 142381 & 25740 & 21280 & 24291 & 46291 \\
\hline 2018 & 166699 & 25484 & 18805 & 17417 & 37190 \\
\hline 2017 & 141319 & 25850 & 19222 & 12863 & 29980 \\
\hline
\end{tabular}

Table 2. Apple sales by regions through 2017-2020 years (Adapted from the annual reports of Apple Inc.)

\begin{tabular}{|c|c|c|c|c|}
\hline region_thous_dollars & 2020 & 2019 & 2018 & 2017 \\
\hline us & 124556 & 116914 & 112093 & 96600 \\
\hline other countries: & 149959 & 143260 & 153502 & 132634 \\
\hline Europe & 68640 & 60288 & 62420 & 54938 \\
\hline China & 40308 & 43678 & 51942 & 44764 \\
\hline Japan & 21418 & 21506 & 21733 & 17733 \\
\hline rest of Asia Pacific & 19593 & 17788 & 17407 & 15199 \\
\hline
\end{tabular}

Microsoft Corporation was founded in 1975 by Paul Allen and Bill Gates. The company sells operating systems, cross-device productivity applications, server application, business solution applications, desktop and server management tools, software development tools, video games, training and certification of computer system integrators and developers. It is headquartered in Redmond, Washington and has offices in more than 190 countries with around 166,475 employees (as for FY2020). Satya Nadella is currently in charge of Microsoft.

Microsoft is one of the leading players in the software industry through its Windows operating system and office utility tools. Its sales of server products and office products along with their cloud services accounted for more than half of total sales in 2020. Microsoft ranks one of the five largest cloud service providers among with AWS, IBM, Oracle and Google.

Unstable cybersecurity and unsuccessful acquisitions like WebTV, LinkExchange, Massive and Danger have been the biggest weaknesses of Microsoft.

768 *Corresponding Author: Prof. Abduraim Vahobov

Volume 04 Issue 07 July 2021

Available at: ijesrr.org

Page No.-767-786 
International Journal of Current Science Research and Review

ISSN: 2581-8341

Volume 04 Issue 07 July 2021

DOI: 10.47191/ijesrr/V4-i7-21, Impact Factor: 5.825

IJCSRR@ 2021

www.ijcsrr.org

Table 3. Microsoft sales by products through 2017-2020 years, in thousand dollars. (Adapted from the annual reports of Microsoft corporation)

\begin{tabular}{|c|c|c|c|c|c|c|c|c|c|}
\hline year & server_products_and_cloud_services & office_products_and_cloud_services & Windows & gaming & Linkedln & search_advertising & devices & enterprise_services & other \\
\hline 2017 & 21649 & 25573 & 18593 & 9051 & 6219 & 2271 & 5542 & 5062 & 2611 \\
\hline 2018 & 26129 & 28316 & 19518 & 10353 & 5259 & 7012 & 5134 & 5846 & 2793 \\
\hline 2019 & 32622 & 31769 & 20395 & 11386 & 6759 & 7628 & 6095 & 6124 & 3070 \\
\hline 2020 & 41379 & 35316 & 22294 & 11575 & 8077 & 7740 & 6457 & 6409 & 3768 \\
\hline
\end{tabular}

Table 4. Microsoft sales by regions through 2017-2020 years (Adapted from the annual reports of Microsoft Corporation)

\begin{tabular}{|c|c|c|c|c|}
\hline regions_thous_dollars & 2020 & 2019 & 2018 & 2017 \\
\hline us & 73160 & 64199 & 55926 & 51078 \\
\hline Other countries & 69855 & 61644 & 54434 & 45493 \\
\hline
\end{tabular}

Figure 1. Apple and Microsoft sales by products for FY2020

Apple sales by products for $\mathbf{2 0 2 0}$

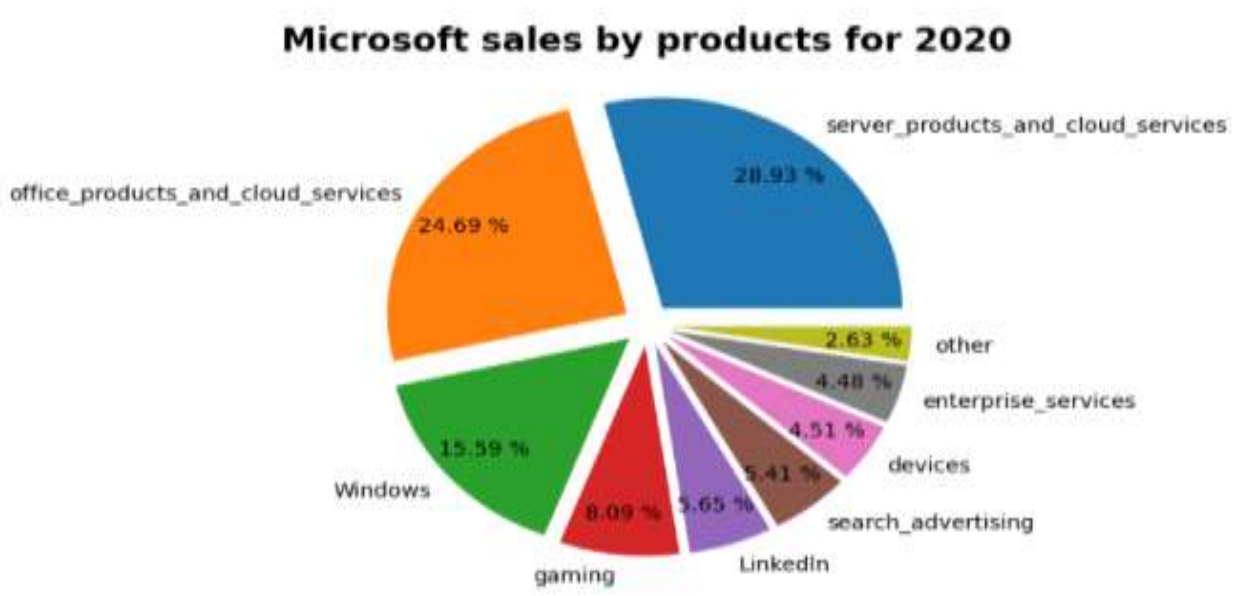

769 *Corresponding Author: Prof. Abduraim Vahobov

Volume 04 Issue 07 July 2021 Available at: ijcsrr.org

Page No.-767-786 
International Journal of Current Science Research and Review

ISSN: 2581-8341

Volume 04 Issue 07 July 2021

DOI: 10.47191/ijesrr/V4-i7-21, Impact Factor: 5.825

IJCSRR@ 2021

www.ijesrr.org

Figure 2. Apple and Microsoft sales by products through 2017-2020 years
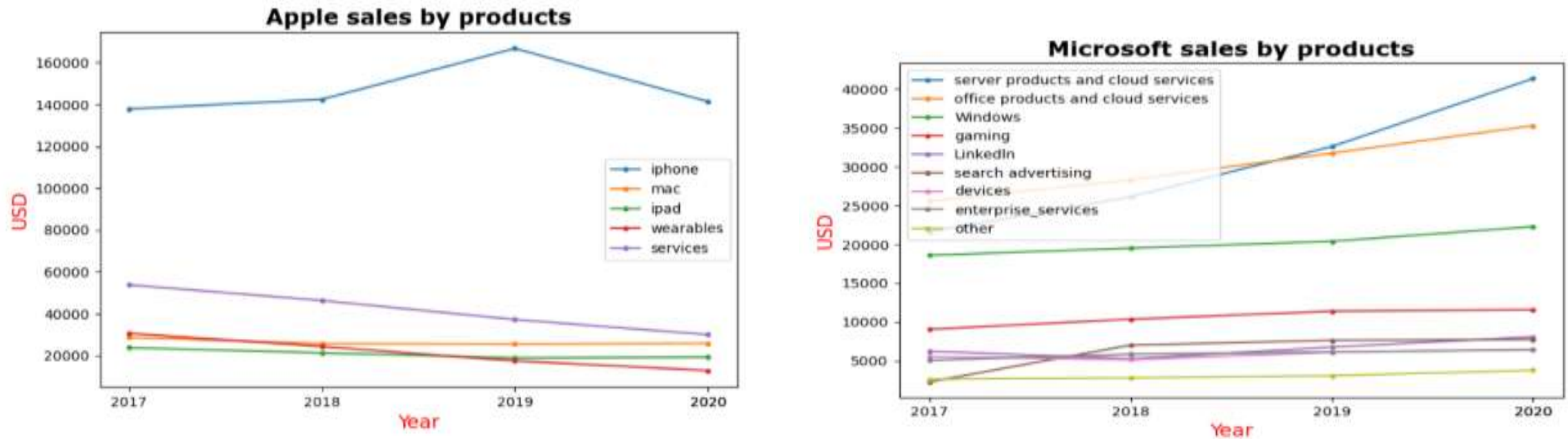

Figure 3. Apple and Microsoft sales by regions through 2017-2020 years

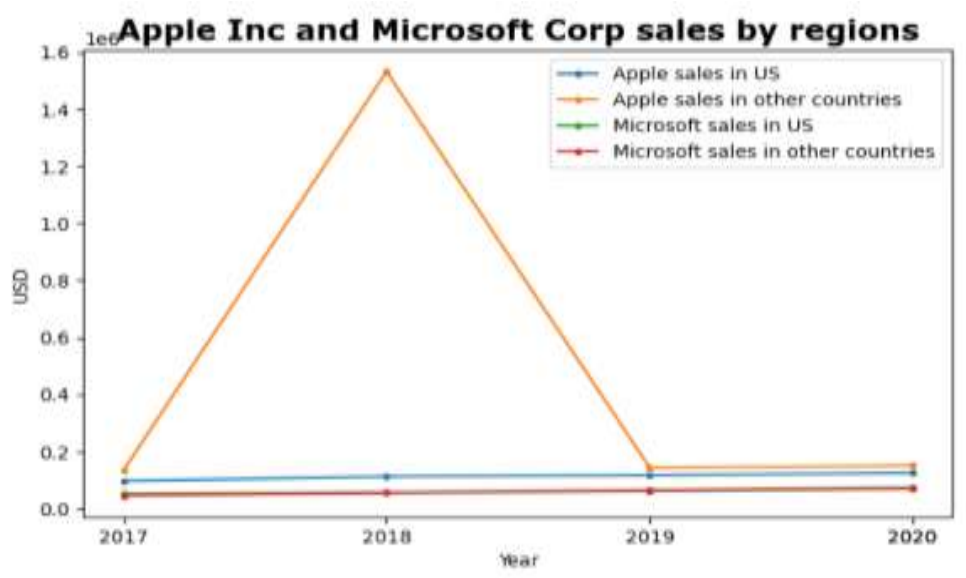

770 *Corresponding Author: Prof. Abduraim Vahobov

Volume 04 Issue 07 July 2021

Available at: ijcsrr.org

Page No.-767-786 
International Journal of Current Science Research and Review

ISSN: 2581-8341

Volume 04 Issue 07 July 2021

DOI: 10.47191/ijcsrr/V4-i7-21, Impact Factor: 5.825

IJCSRR@ 2021

www.ijesrr.org

From 2017 to 2020 Microsoft sales were equally distributed to both US and other countries, while Apple had been highly selling to other countries by 2019 .

Table 5. Apple and Microsoft sales forecast for 2021-2025

\begin{tabular}{|l|l|l|}
\hline year & APPLE & MICROSOFT \\
\hline 2017 & $\$ 229,234,000,000$ & $\$ 89,950,000,000$ \\
\hline 2018 & $\$ 265,595,000,000$ & $\$ 110,360,000,000$ \\
\hline 2019 & $\$ 260,174,000,000$ & $\$ 125,843,000,000$ \\
\hline 2020 & $\$ 274,515,000,000$ & $\$ 143,015,000,000$ \\
\hline 2021 & $\$ 257,379,500,000$ & $\$ 117,292,000,000$ \\
\hline 2022 & $\$ 264,415,875,000$ & $\$ 124,127,500,000$ \\
\hline 2023 & $\$ 264,121,093,750$ & $\$ 127,569,375,000$ \\
\hline 2024 & $\$ 265,107,867,188$ & $\$ 128,000,968,750$ \\
\hline 2025 & $\$ 262,756,083,984$ & $\$ 124,247,460,938$ \\
\hline
\end{tabular}

In table 5, sales of both companies were extrapolated with 5-period moving average till 2025, assuming, that sales keep same growth trend.

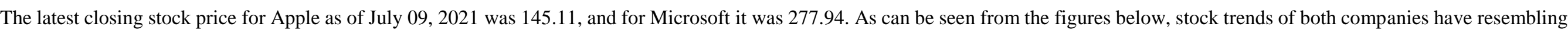

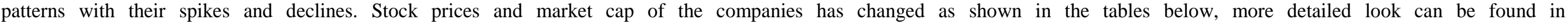
https://github.com/Muniska/Project. 
International Journal of Current Science Research and Review

ISSN: 2581-8341

Volume 04 Issue 07 July 2021

DOI: 10.47191/ijesrr/V4-i7-21, Impact Factor: 5.825

IJCSRR @ 2021

www.ijcsrr.org

Table 6. Apple and Microsoft stock price trends through 2017-2021 yy.

\begin{tabular}{|c|c|c|c|c|c|c|c|c|}
\hline & date & open & high & low & close & volume & average_price & market_cap \\
\hline 0 & $03 / 01 / 2017$ & 27.3275 & 27.4526 & 27.0821 & 27.4101 & 115127460 & 27.26735 & 3155.655191 \\
\hline 1 & 04/01/2017 & 27.3393 & 27.4951 & 27.3157 & 27.3795 & 84472464 & 27.40540 & 2312.813828 \\
\hline 2 & 05/01/2017 & 27.3559 & 27.5787 & 27.3299 & 27.5187 & 88774348 & 27.45430 & 2442.954650 \\
\hline 3 & $08 / 01 / 2017$ & 27.5588 & 27.8845 & 27.4857 & 27.8255 & 127007600 & 27.68510 & 3534.049974 \\
\hline 4 & $09 / 01 / 2017$ & 27.8348 & 28.1842 & 27.8326 & 28.0803 & 134247792 & 8.00840 & 3769.718274 \\
\hline$\ldots$ & & & & & & ... & $\ldots$ & \\
\hline 1115 & $09 / 06 / 2021$ & 127.2100 & 127.7500 & 126.5200 & 127.1300 & 55584187 & 127.13500 & 7066.417693 \\
\hline 1116 & 10/08/2021 & 127.0200 & 128.1900 & 125.8400 & 126.1100 & 70606505 & 127.06500 & 8904.186346 \\
\hline 1117 & 11/08/2021 & 128.5300 & 127.4400 & 128.1000 & 127.3500 & 52839952 & 126.77000 & 6729.167887 \\
\hline 1118 & $14 / 08 / 2021$ & 127.8200 & 130.5400 & 127.0700 & 130.4800 & 96906490 & 128.80500 & 12644.358815 \\
\hline 19 & 5/006/2021 & .8400 & 8000 & 3900 & 6400 & 60771138 & 129.99500 & 7878.370071 \\
\hline
\end{tabular}

1120 rows $\times 8$ columns

\begin{tabular}{|c|c|c|c|c|c|c|c|c|}
\hline & ate & en & gh & w w & lose & volume & iver & $\operatorname{mar}$ \\
\hline 0 & 1/2017 & 58.8000 & 3.8475 & 8.1778 & 58.6040 & 20694101 & 1270 & 1212.757 \\
\hline 1 & 01/2017 & 51 & 7632 & .1732 & 3418 & 21339969 & 46820 & 1245. \\
\hline 2 & 05/01/2017 & 2388 & .6789 & 0889 & 3418 & 875968 & 90 & 750 \\
\hline 3 & $06 / 01 / 20$ & 3418 & 1378 & .0983 & 3.8475 & 19922818 & 805 & 976 \\
\hline 4 & 2017 & 7725 & 0722 & 5665 & 6802 & 82730 & 1935 & 018 \\
\hline$\cdots$ & ... & ... & $\cdots$ & $\cdots$ & ... & ... & $\ldots$ & \\
\hline 15 & $08 / 06 / 2021$ & 253.8100 & 255.5300 & 253.2100 & 253.5900 & 17873668 & 8.37000 & 4532.583468 \\
\hline 1116 & 10/00/2021 & 254.2900 & 257.4600 & 253.6700 & 257.2400 & 32 & 00 & 6282 \\
\hline 1117 & $11 / 06 / 2021$ & 257.9850 & 258.4868 & 256.6100 & 257.8900 & 18791793 & 257.54840 & 4846.215497 \\
\hline 118 & 14/06/2021 & 8000 & 00 & 00 & 00 & 0507 & & 4977.025264 \\
\hline 1119 & 6/08/2021 & 9.7750 & .9900 & 7.6800 & 258.3600 & 034100 & 258.83500 & 4400.93007 \\
\hline
\end{tabular}

Figure 4. Apple and Microsoft stock market development visualizations for 2017-2021 yy.

AAPL stock price candlestick visualization

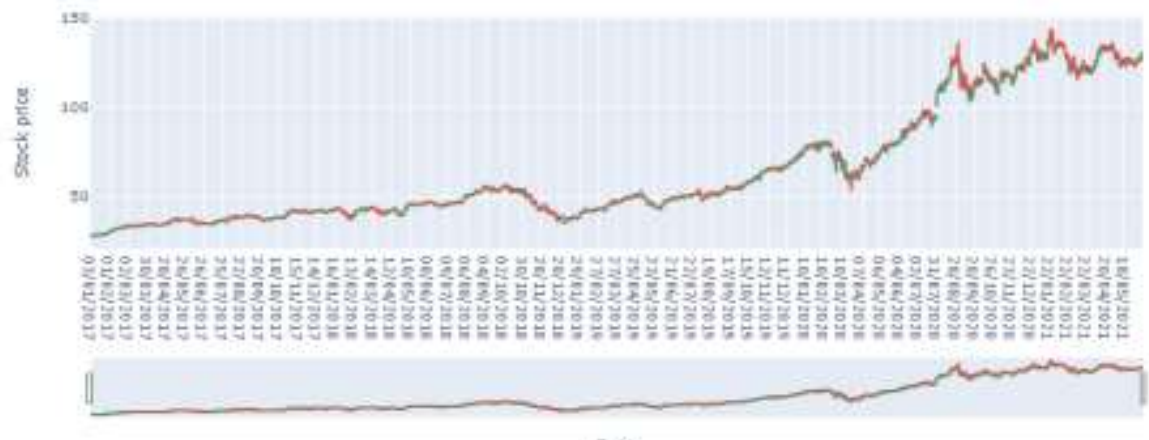

Dote
MSFT stock price candlestick visualization

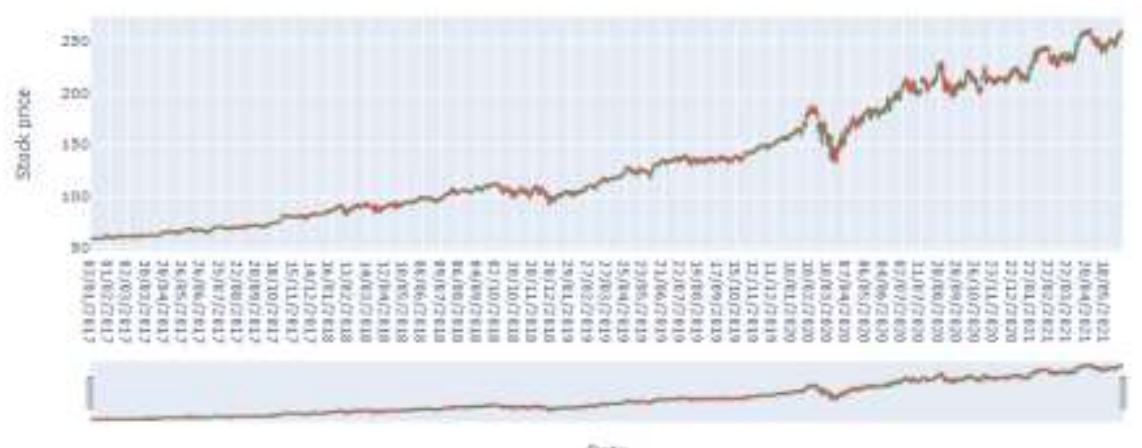

pote 
International Journal of Current Science Research and Review

ISSN: 2581-8341

Volume 04 Issue 07 July 2021

DOI: 10.47191/ijesrr/V4-i7-21, Impact Factor: 5.825

IJCSRR @ 2021

www.ijesrr.org
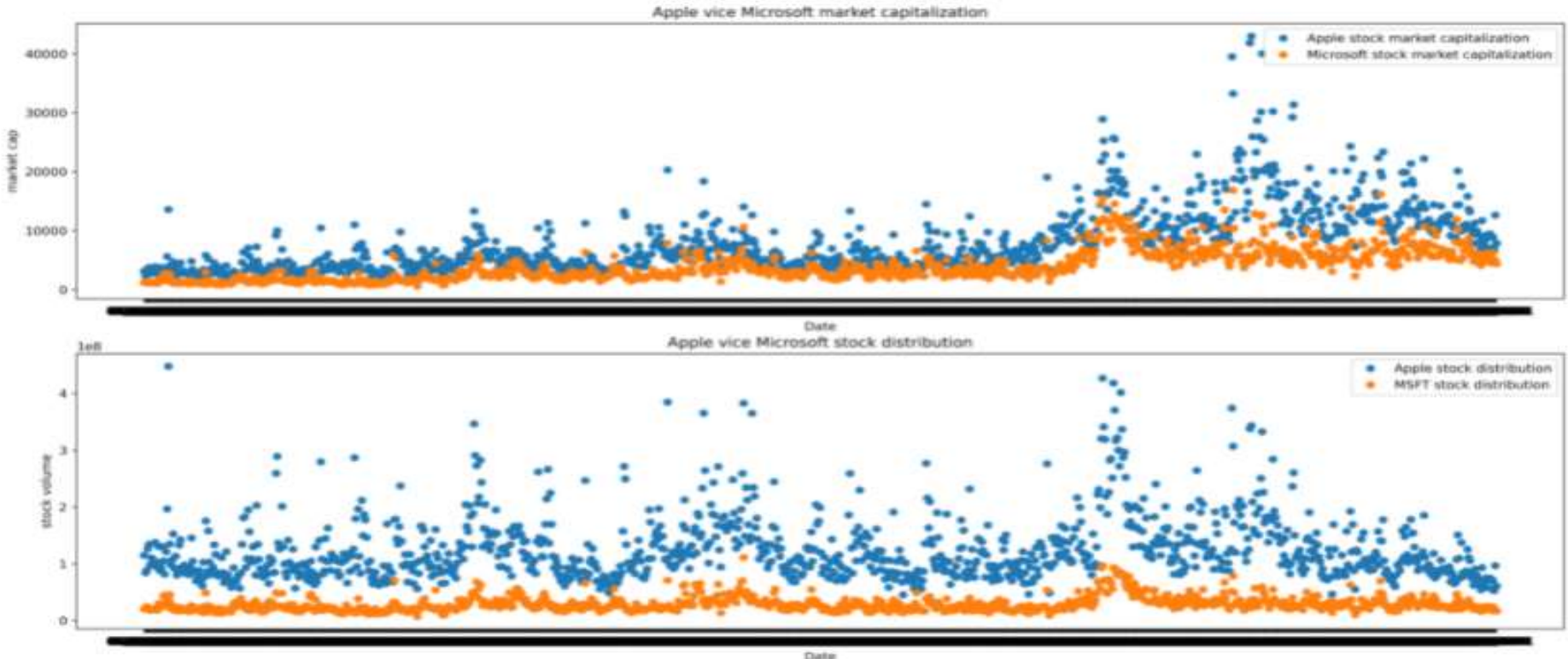

\section{INCOME STATEMENT ANALYSIS FOR 2017-2020}

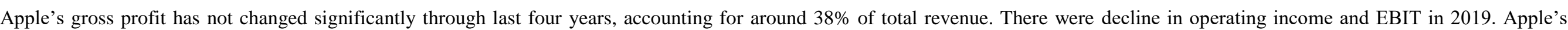
earnings per share have been rising from $\$ 2.32$ to $\$ 3.31$.

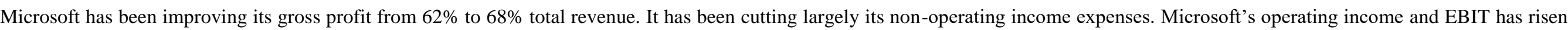
for around $20 \%$ in 2019 . The company's earnings per share increased from $\$ 2.74$ to $\$ 5.82$. 
International Journal of Current Science Research and Review

ISSN: 2581-8341

Volume 04 Issue 07 July 2021

DOI: 10.47191/ijcsrr/V4-i7-21, Impact Factor: 5.825

IJCSRR @ 2021

www.ijesrr.org

Table 7. Apple Inc. Income statement vertical and horizontal analysis (Adapted from the annual reports of Apple)

\begin{tabular}{|c|c|c|c|c|c|c|c|c|c|c|c|c|c|c|}
\hline netenter Is & montrasa & sertentagerasoso & meumzars & Derceptapezalo & Amenesois & 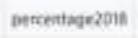 & moantars & gencertagesion: & engeros: & 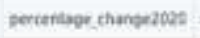 & Amototints & 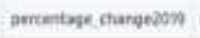 & inanoctols & ontentage chanpezeut \\
\hline tescyentua & 1274515,0000003 & 500000 & 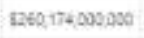 & 1000000s & DEES 305,005002 & 1000006 & $2229,2340000,000$ & 1500005 & $514 ;=1900,502$ & $35 \mathrm{rs}$ & 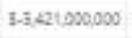 & 2045 & $136: 3650023050$ & $15896 \%$ \\
\hline ostolutitose & S169,599,000,002 & $61, m$ & ste1,782.0320se & 62185 & $5162,756,000,002$ & 51.6655 & 347,:40,0000,000 & 81,395\% & st,m,0ssoco & 4035 & 3.194/,050000 & -1245 & 522,0040000000 & $16,10 \%$ \\
\hline grous_iport & 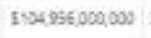 & $3823 \mathrm{~s}$ & 991,3220000030 & 37825 & F10(a39,002,002 & 30205 & sas, , Be,0020050 & $3 E 4 \pi 5$ & 36,564080050 & $687 \mathrm{v}$ & 5-3.347,050000 & -3.005 & $\$ 13653,000,020$ & 154865 \\
\hline Seliegeners grd gshintizbie & 559916,000900 & 7295 & 110245000000 & 7aा & See 705,0500000 & 6396 & 515261,000000 & 666x & 51,651050000 & $\operatorname{sins}$ & 11.5ec0000000. & $\sin$ & 1144.5005000 & Fats \\
\hline Aetescrondfereapnert & 5187825000900 & $\cos 8$ & s1e20,000000 & $\cos$ & 11e20,0200000 & 3364 & 31135.5020000 & $205 \%$ & 12,2510000000 & thess & $5196+002028$ & 11925 & 12.635200500 & $22 \mathrm{~ms}$ \\
\hline 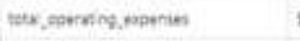 & 538660,000000 & 4005 & $324+2: 200035$ & 1325s & $590 \mathrm{sut}+050000$ & $1+\operatorname{tes} 5$ & 52.,0425020000 & 13.715 & H4,25eass050 & 12200 & 53,521000000 & 10398 & 34099603005 & $152 \pi 5$ \\
\hline epernirg, mante & $556.2080000,000$ & $26.15 \mathrm{~s}$ & 3619300001000 & 24.575 & $50,698050,000$ & $25 \cos$ & 151346001000 & $26.78 \mathrm{~s}$ & 18,15a, ass scee & 2eas & $1-690000000$ & atass & $1592 \times 5001500$ & $1929 \%$ \\
\hline 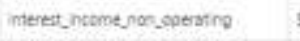 & $537 \pm 2000000$ & us & $5496,000,500$ & 1915 & 15,6650000000 & 1245 & $15221,000,0008$ & 2275 & 5. 1, 198000000 & .26155 & $1-725,0000000$ & -12795 & 5450050000 & 5.395 \\
\hline intret, espenst, ran, osterting & $12,673,0000058$ & 1095 & Fast 000,000 & 1.178 & 532400003030 & $121 \mathrm{~s}$ & $122,329000,005$ & $10 \mathrm{sta}$ & 5.703,0000000 & $.1260 \mathrm{~s}$ & $3598,000,000$ & 10.575 & 58178000050 & $90,4 \%$ \\
\hline 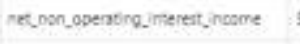 & sssac0020000 & 0.25 & $51,305,000,000$ & 0.395 & $522,46,5020200$ & 0925 & $\$ 2,972,000,000$ & 12es & $5,-45,0020000$ & $-35724 \mathrm{~s}$ & $s=10610500000$ & -43365 & $5.432,000,000$ & -16048 \\
\hline 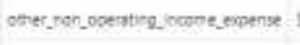 & $597,000,000$ & $\cos s$ & 1020000009 & a.1en & $\operatorname{ser}(000000$ & 0.15 & 1ust,0000000 & a.ses & 5-535,900,050 & 793006 & 1-19,050,000 & $43 \div$ & 15000000000 & meses \\
\hline suribjiftares & ST:00:000,000 . & $24.45 \mathrm{~s}$ & 365737000005 & s2ns & $172,023000,000$ & $27,45 \mathrm{~s}$ & K4+0090000050 & 27:ses & 51 $15+050000$ & 2005 & 4.7, $140,050,000$ & .4835 & 548.45003050 & 1395 \\
\hline tasprovisen & $59,830,500,000$ & $359 \mathrm{~s}$ & 150,41,000,035 & 6035 & $513,72,080,000$ & 5.335 & I157360000000 & GB7s & 4-801,002030 & .7846 & $5-2091000000$ & -215155 & $5-2,366,9020000$ & -15935 \\
\hline 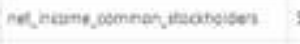 & $5974+1+0000500$ & $20 \times 5$ & 155,256,000,025 & 21245 & 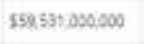 & $22+15$ & 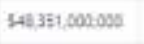 & $21.00 \mathrm{~s}$ & 52,1550989050 & 3005 & $1-42750000000$ & $-7+25$ & 571,1830000030 & H124 \\
\hline Descefps & 533! & & 1209 & & 1320 & & 1232 & & $\operatorname{san} 2$ & 10606 & 5-001 & 24,45 & 5068 & 29635 \\
\hline ainiteojers: & 5328 & & ne97 & & 1298 & & 8203 & & 1000 & 12320 & 1000 & A12\% & 1207 & 1950s \\
\hline incicuenseytrots & t738213900s & & $\operatorname{tentusy0500}$ & & 196215900000 & & soessoresos & & 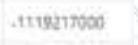 & reses & -13500772056 & $-\sin 5$ & -10474520000 & -5 aes \\
\hline Gilad, prenge, tham & 17528244003 & & 165setseso & & 2000536050 & & 2rooptesicos & & -1067450000 & $.5,7.45$ & 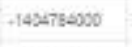 & -7025 & -1056382030 & ATS\% \\
\hline 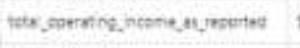 & seczeasoosos & 2t.1ss & ies999000035 & 2495 & 670, 208080000 & zeess & 561.34+505000 & zaters & 12.2se ossoce & $369 \mathrm{~N}$ & $1-4968050000$ & -4.935 & 95954503500 & $155 \mathrm{~m}$ \\
\hline sexe-enperses & 5200227000000 & 7345 & 696244009900 & $75+35$ & (194 का? 500002 & tars & 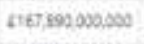 & 73245 & 21, 1930500009 & sive & $31,547,0000000$ & args & $126,007,0000,000$ & 1595 \\
\hline narmvices nnesme & $527,41,000,300$ & $20 \% 15$ & $159,256,000,000$ & $2 t 246$ & 1595250000000 & 2245 & [AB: 351,003500 & 21095 & 52,15: 0000000 & 2005 & $3+275050000$ & - 2ias & 31,402005030 & 19124 \\
\hline interest mooms & 53702,000000 & 395 & EAsel 000,000 . & t.915 & :5, 666,005050 & 2.145 & Es $201,000,000$ & $227 \mathrm{~s}$ & S-1,195,000,000 & $.2415 \%$ & 5-725000,000 & -12758 & satas por 0000 & 3.325 \\
\hline interestesserse & 1230,000000 & rass & DSste0s0000 & ars & $53,240,003050$ & 1205 & $62 m 000000$ & 1015 & 5709000050 & $49 \mathrm{ens}$ & 1386,000000 & $103 \pi$ & 39170302000 & 1907 \\
\hline ne interatipeons & tonosonose & easts & 61,3850s00000 & a.59s & $12,46,008,050$ & 0.924 & 62.8710009009 & t285 & 5. Ans,osososo & sam & Lton, $, 000,000$ & Asses & 1.458,000,000 & thots \\
\hline thit & Mag,9ta,000,0000 & 2406 & ite:318,000,050 & ses: & i26, $163,050,000$ & 25675 & $646,472,002090$ & $2 \mathrm{~kg} 5$ & $365: 050,500$ & ars & $1-40500000000$ & 295 & $18,731,000,000$ & 1645 \\
\hline 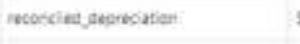 & $511,056,000,200$ & 4295 & [12507,000,0090 & $4: 25$ & [12,90:0906000 & 4स: & \$19,157, & 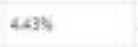 & I- $-425,000,000$. & .14185 & $51,64+000050$ & 15985 & $15+202030$ & 7345 \\
\hline 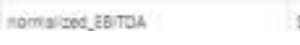 & $251,000,000,900$ & $29: 916$ & FEtaed ocq oss & 31485 & : $657,0<5080,000$ & $32 \pi 5$ & $276,599,002050$ & 33456 & 3-540,000,000 & tase & 1-5:106,000,000 & -5.966 & $-519+470202030$ & 136656 \\
\hline
\end{tabular}


International Journal of Current Science Research and Review

ISSN: 2581-8341

Volume 04 Issue 07 July 2021

DOI: 10.47191/ijesrr/V4-i7-21, Impact Factor: 5.825

IJCSRR @ 2021

Www.ijcsrr.org

Table 8. Microsoft Income statement vertical and horizontal analysis (Adapted from the annual reports of Microsoft Corp.)

\begin{tabular}{|c|c|c|c|c|c|c|c|c|c|c|c|c|c|c|}
\hline inscitat is & amoum2020 & peticentraperase & ameunt2e19 & percentage20!9 & snounteols & Dersentage2018 & Amount2017 & Dencemaqezon? & clange2020 & Dercenthose,change 2020 & damee2019: & Defcentage orangezo19 & change2er18 & peritentuge change2018 \\
\hline totes reverve. & stes:019,000:000 & 1920005 & $5123,64,000,003$ & $100000 \%$ & $3110,360,000,000$ & $100000 \mathrm{k}$ & $568,950,0002000$ & 1000000 & $517,172,000,000$ & 13655 & $515,469,050,000$ & 14034 & $120,410,002,000$ & 22.695 \\
\hline 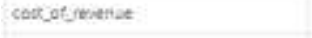 & $\$ 26,078,003,503$ & 32215 & $3,2,2,10,000,950$ & $34.10 \mathrm{~s}$ & 138,353:000000 & 34.758 & $53,121,000: 000$ & 360998 & $83,60,000,000$ & 2300 & 5,537,000,000 & $1200 \%$ & $54,092,0000,000$ & 1196\% \\
\hline giossporth & 396,937,000,003 & 62785 & $382,933,0000250$ & $65900 \mathrm{~N}$ & अ2:007,000,000 & $65.25 \%$ & $865,669,002,000$ & et9:\% & $\$ 14054,000,000$ & 16005 & $510.9260000,090$ & 75,775 & 516,9Ta,900,000 & $2020 \%$ \\
\hline 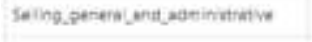 & $524,00,000,000$ & 17255 & 124:0n 050,050 & 14955 & 522222050903 & 20165 & 520000000000000 & $222 \mathrm{es}$ & $5 \times 511,000,000$ & $\cos 5$ & 10750002,000 & 3945 & $12,201,000,000$ & 11005 \\
\hline 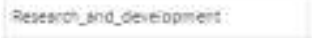 & $519,299,005,000$ & 13.475 & $51 \leq 376,0000030$ & 13418 & 5s4726,0000003 & 13.345 & 813,937,0070000 & Le95: & $32,383,0000000$ & $3,406 \%$ & $\$ 2,150,008,000$ & $1460 \%$ & $51,659,000,000$ & 129606 \\
\hline 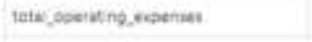 & S43.v78,000,000. & torss & 196s? 0000000 & $31,78 \mathrm{~s}$ & 13E.940.0000025 & 33,85 & $131,052,000,000$ & 36795 & 540040000000 & saoss & 33,2025,005:0025: & 8.95 & $\$ 3,8022,000,000$ & 11778 \\
\hline astrane noome & 532999,0009000 & 37.035 & $522959,000,050$ & $34.10 \mathrm{~s}$ & 3350560000000 & $3: .7 m$ & 322.532 .000000 & 25.16\% & $50000,000,000$ & 23205 & 17,sot gos.aso & 223as & $12,40.900,000$ & $9605 \%$ \\
\hline \multicolumn{15}{|l|}{ 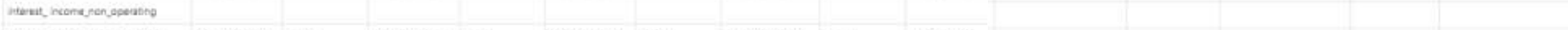 } \\
\hline 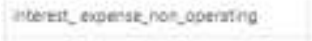 & $52,591,080000$ & $13+\mathrm{s}$ & $32,6 a 6,005,008$ & $213 \%$ & 52733000000 & 24as & $122222,000,000$ & $2 \operatorname{sen}$ & 5.95,000,000 & ssess & $1-47,000,000$ & +1.225 & 5512000,000 & 25025 \\
\hline 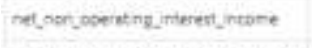 & $12.295000,000$ & tasts & 3.t.64t cosasa & 2158 & 3.2735000000 & 2455 & $1-2+22000000$ & 2075 & $150,000,000$ & .3546 & 347,0009000 & .1 .726 & $5-515,502,000$ & $2500 \%$ \\
\hline otrey, $n$ on, aseristing income_ergense & 22660050050 & ta7s & $53,415,000,005$ & 2715 & $34: 45000,000$ & 3760 & 5:310000,000 & $0.15 \mathrm{~N}$ & 5.747,000,000 & 21.875 & 5.734,000,0000 & $-7769 \mathrm{~s}$ & $5<, 016,000,000$ & $30 \times 9.558$ \\
\hline 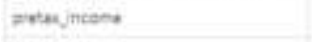 & 552090,0000000 & 37.045 & S4se64050,050 & 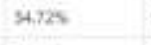 & $296674,000,005$ & sass & 523,141000000 & $25,7 \mathrm{es}$ & 58540000050 & HaDs & $15.214000,050$ & 19.7285 & $3+3385,900,050$ & 57565 \\
\hline taugravisen & 38759050000 & 6.120 & $3<445,008,800$ & 3598 & J19.90s,000,800 & 10055 & 11,945000000 & $2 \mathrm{~ms}$ & 34 307,000000 & 96005 & 1-15,455,000000 & $\pi, 003 \mathrm{~s}$ & $177,910.000,000$ & $983.2 \mathrm{~m}$ \\
\hline 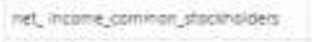 & $566201,090,900$ & In 965 & $599,200000.580$ & 31. & $310,575,000,500$ & $15 a 25$ & 521204000000 & $23,5 \pi$ & 3130000000 & 12855 & 212:t69,050,000 & 736.805 & $1-4693000,000$ & -21.095 \\
\hline sask,ges & $55 a_{2}$ & & sE:11 & & $12+15$ & & 5274 & & \$1 & 13700 & ss & 135030 & 3-1 & -21,:3v \\
\hline divestes & $55: 76$ & & $3 s \infty 0$ & & s213 & & $52 \pi$ & & s1 & $19 \sin$ & 53 & $13805 \mathrm{~s}$ & 5-1 & $-21,470$ \\
\hline ariculanceshures & 7510000050 & & $7 \in 79000000$ & & 7000000000 & & 7746500000 & & $5.63,005,000$ & $.0 .82 \mathrm{~s}$ & $5-27,000,000$ & -a.35s & 5.-460000050 & -osen \\
\hline dintos,newgeram & 7855000000 & & 779000000 & & 7704000000 & & 7855005050 & & L 2000050000 & s.90s & $1 \rightarrow+000000$ & .0535 & 5.92000,005 & aaps \\
\hline 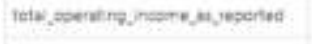 & $352.250,000,000$ & 37015 & H2OSP 050 osto & 3्र.125 & $335048,000,005$ & $\operatorname{sins}$ & $522+12002,00009$ & isies & 120,050,000,000: & $2328 \mathrm{~K}$ & $57,000,000,050$ & 22.545 & $5+2,216,000,000$ & 5.90\% \\
\hline foto_endecses & $599,055,000,000$ & 62978 & $562 B 640000,050$ & 65.965 & $575,302,000,000$ & 60235 & 567. 31000200000 & 74806 & 52172,000050 & sess & $57: 2 \times 2000,090$ & 10075 & 57,984050005 & tratses \\
\hline 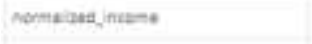 & S4t2ar 9000000 & $3996 \mathrm{~s}$ & $512,240050,050$ & $3 r, 165$ & $516,571,000,003$ & $15 \mathrm{~ms}$ & 521,20400200000 & 23.578 & $5504+000,090$ & 12085 & $312,669,000,000$ & 136.000 & S-4653,000,000 & -21050 \\
\hline \multicolumn{15}{|l|}{ rteert nosme } \\
\hline pherentegense & $52,59+, 000,050$ & $14+5$ & $32,686,000,909$ & $213 \mathrm{~s}$ & $12733,000,050$ & $2,43 \mathrm{~s}$ & $52,22,000,000,00$ & 2475 & 5.950000000 & $.33+5$ & 1-47,0050000 & $-1,726$ & $5511,000,000$ & 23.025 \\
\hline inet ntevertincosere & $1-2,591,000,000$ & .7815 & $1.2006,900000$ & .2 .198 & 12735000,000 & 2485 & 5-2.222.902000000 & $2 \pi s$ & s59,0090000 & sass & S47,000,000 & $1,72 \%$ & 1-310005000 & 25as \\
\hline tarr & s5rezt,000,000 & 38005 & HeS374050 000 & sesss & Fas 2007,000000 & 75935 & 225.37,000,000.00 & ands & 59.259000000 & 1995 & 81, 167000,000 & 18.265 & $\$ 13: 936000,050$ & ses9s \\
\hline reconcies,desteciation & $512796,000,000$ & 895n & 31: 682000050 & 92856 & 310261,002000 & 9.300 & s8 $778,050,000000$ & 9.755 & : : $: 1140000000$ & Fers & 11,410005000 & 1395 & $51,401,000,000$ & 16.195 \\
\hline 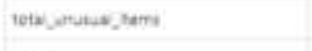 & 52700050030 & 1.906 & $13+22,000,500$ & 2759 & $1+200,050,050$ & 3.9.5 & $12.070000,03000$ & $33: 5$ & 4. $-564050,050$ & .220005 & E. $736,050,000$ & $-1749 \mathrm{n}$ & 51.2180000000 & 42745 \\
\hline nomerised_tertou & 1es,7ns,0005000 & 45998 & 154,3840000000 & .43.370 & $545,260,0000000$ & thers & $131,159,000,00000:$ & 3ekess & $35,131,000,000$ & 20395 & $39,244000,000$ & 22605 & 514.15t, 2000,000 & 45258 \\
\hline
\end{tabular}

775 *Corresponding Author: Prof. Abduraim Vahobov

Volume 04 Issue 07 July 2021

Available at: ijcsrr.org

Page No.-767-786 
International Journal of Current Science Research and Review

ISSN: 2581-8341

Volume 04 Issue 07 July 2021

DOI: 10.47191/ijcsrr/V4-i7-21, Impact Factor: 5.825

IJCSRR@ 2021

www.ijesrr.org

Figure 5. Apple and Microsoft income statement visualizations for 2017-2020
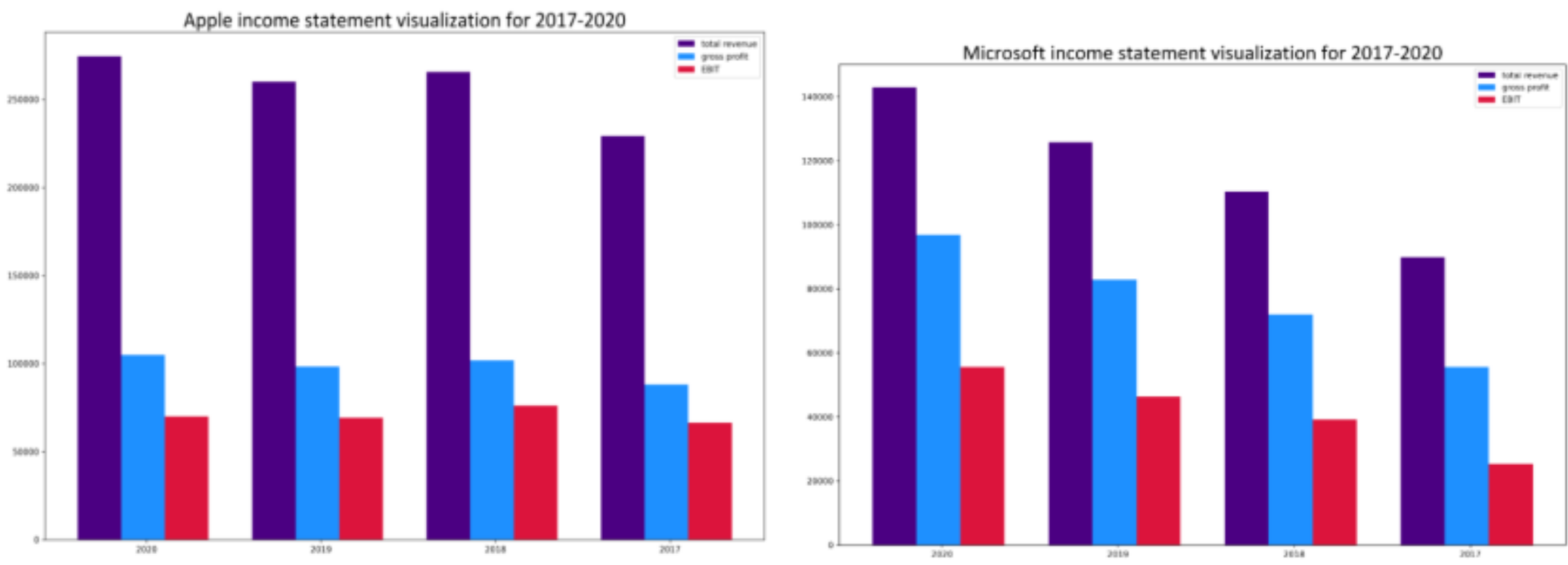
International Journal of Current Science Research and Review

ISSN: 2581-8341

Volume 04 Issue 07 July 2021

DOI: 10.47191/ijcsrr/V4-i7-21, Impact Factor: 5.825

IJCSRR @ 2021

www.ijcsrr.org

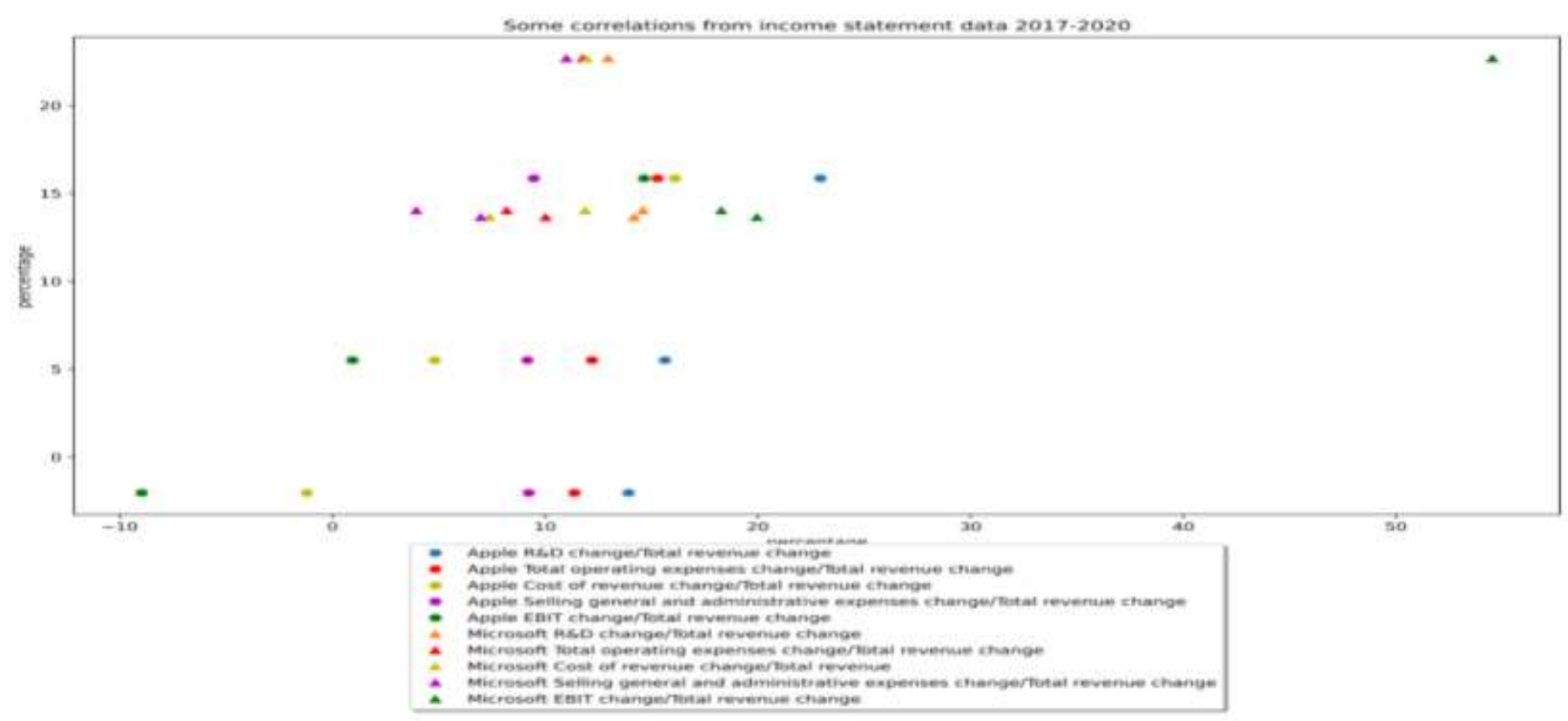

\section{BALANCE SHEET ANALYSIS FOR 2017-2020}

Apple's assets had been decreasing from 2017 to 2020 . If in 2017 total current assets of the company accounted for $34.28 \%$ total assets, in 2020 it reached $44.37 \%$ of total assets. However total liabilities also surged in 2018 and 2020 years.

Microsoft's assets on the contrary were increasing from 2017 to 2020 . Total current assets of the company accounted for $66.30 \%$ of total assets in 2017 , which plumped to $60.37 \%$ in 2020 . Although total liabilities had not changed over time, its share in total assets had been less than that in 2017. 
International Journal of Current Science Research and Review

ISSN: 2581-8341

Volume 04 Issue 07 July 2021

DOI: 10.47191/ijcsrr/V4-i7-21, Impact Factor: 5.825

IJCSRR@ 2021

www.ijesrr.org

Table 9. Apple Inc. Balance sheet vertical and horizontal analysis (Adapted from the annual reports of Apple)

\begin{tabular}{|c|c|c|c|c|c|c|c|c|c|c|c|c|c|c|}
\hline Edicator, as & seoumzozo & percentage2020 & amountzov9 & Dercernoezong & mosumases & pescentagezovs & mousanit & Dercentspezors & arengezaso & pencentage change2020 & changez:orto & sertentage changezers & chespezo18 & pestembege thangezeri \\
\hline Totre, aness & 5323:858 0000000 & 100005 & $\$ 338,516,000,900$ & 102005 & 5365, J25,000,030 & 1000005 & \$372319,002000 & $150000 \%$ & 5. $14,229,000,002$, & As & $5-27,209,902005$ & taes & 5.9996000,000 & 2.5es \\
\hline 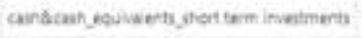 & silemi:0005000. & 20005 & $5100,557,050,505$ & 20714 & $36 \in 300,050,005$ & 14138 & $57+1860000000$ & 0.765 & $5-864,900050$ & .105 & 534,25e,000,90a & \$1⿴囗十 & $5-7.36009000000$ & $.052 \mathrm{~s}$ \\
\hline recenabies & 537,45000000 & $11: 5 e$ & 545886005050 & 13530 & \$4 $45996,050,0000$ & 15020 & 135,6750500000 & 5980 & 3.4398002050 & .165 & 5.3191,000,000 & .6515 & s13522,0000,0000 & s73as \\
\hline mariby & $34,56 \times 000,500$ & 1295 & $5+106050000$ & 1215 & $53956,000,000$ & toses & 4255000,000 & $12 \mathrm{~s}$ & $5-450000000$ & .15 & S1500000000 & 3.795 & 5-699,000,000 & -18525 \\
\hline 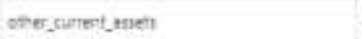 & 51,2540000050 & sats & 1123520050000 & sess & $112007,050,000$ & 395 & 11,954,050000 & $\sin$ & 1.7, 00850000000 & .95 & 52650000000 & 2,195 & 5:1,948,0000000 & .2325\% \\
\hline tos:carrem,assen & $5143,713,0000000$ & $4<37 \mathrm{~V}$ & 8162,819,000,000 & 4a:100 & 581, 359,000000 & $3591 \%$ & II2064, & 34265 & S.19,006,000,000, & $.12 \pi$ & ssi 4600000000 & $23.97 \mathrm{~s}$ & $52,694,000,000$ & 2095e \\
\hline netppe & nae $76 t, 000,000$ & 11:35s & $537,378,005,000$ & 1hows & \$21,354,000,002 & 11295 & $\$ 33,793,900,000$ & 9050 & i. 612000,000 & se & $1-3,926,000,090$ & -2515 & $57,521,000,000$ & $2226 \mathrm{~s}$ \\
\hline 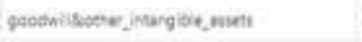 & s. & $0.0 \%$ & s. & 2005 & \&. & $0.00 \%$ & Hsors.000000 & 2.145 & 3. & & s. & & 5.6:15,000000 & . $10000 \%$ \\
\hline 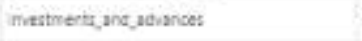 & 51020670000000 & $31.19 \mathrm{~m}$ & 5105321,009000 & 3t. $22 \mathrm{~s}$ & 5170799000000 & $\Delta 6.79 \mathrm{n}$ & II94 Ti4,20050000 & 51385 & $5.4454,0000000$ & .4s & 565.458005005 & -38394 & 522915000000 & $.12225 \mathrm{~s}$ \\
\hline 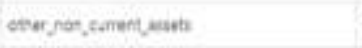 & $14 \div 322000000$ & 13,139 & 932970,008000 & 8.745 & 522283050000 & $600 \times$ & 510162050005 & 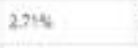 & in:5+4000,008 & 298 & 510.695,002,000 & 40.005 & $512,22,000,050$ & 199255 \\
\hline 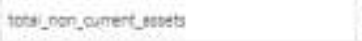 & $5182,175,0059000$ & $25 e x$ & $5173,87,0000000$ & 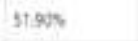 & $524,486,000,050$ & Go5s & 524.6740000000 & satss & 54,400000003 & $3 \mathrm{~s}$ & 1.58 659,000000 & -25.045 & 5.12:288002020 & $4.96 \%$ \\
\hline toth yabities: & s235, 549,000,900: & 79838 & 5240,020, 050,003 & 73275 & $5278,576,000,000$ & 70,786 & $524: 272000000$ & 64365 & $510521,000,050$ & $4 \mathrm{sin}$ & $2-70,550,000,000$ & $.400 \mathrm{~s}$ & $537,300,0000050$ & 7.125 \\
\hline oxpsoes & $542296,000: 000$ & 9005 & $546,236,000,000$ & 13605 & 5S5, acea, 000,002 & $1520 \%$ & $549,069,050,000$ & 33005 & E.3942,003,0000 & .95 & 5.9652000050 & .17275 & seasosspos & ngos: \\
\hline 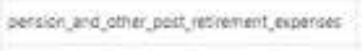 & 5. & 0.005 & s. & 2006 & + & $\alpha 000$ & $\$ 25,74,950,000$ & Esex & s. & & 5. & & 5. $24,744,000,900$ & +03006 \\
\hline surem geat & $513>60000000$ & 4235 & $5162+4005050$ & 4805 & $22274 a 090.909$ & 3675 & 588,75990000 & 2.025 & $3.2271,902080$ & $.15 \%$ & $5-4500900,900$ & $.21,738$ & $22,275,000,000$ & $12.32 \mathrm{~s}$ \\
\hline 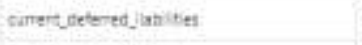 & $30,41050,500$ & $205 \%$ & 18:52000000 & 1ass & 59.930000000 & 2005 & $17560,000,000$ & 2015 & 51, $12+080009$ & 205 & $5-2,021,000,050$ & $26 \% 9 \%$ & 5:5000,000 & .0075 \\
\hline operjounertianites & 522564000008 & 12105 & $587,722,002000$ & 111.146 & 512017000,000 & assis & 5. & 0.085 & 54564000000 & $13 \%$ & 55,033,000,003: & 15.405 & $\operatorname{si2} 687,000,050$ & \\
\hline 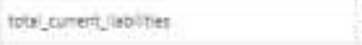 & $5105,392000,000$ & 2250 & s108710.900000 & rass & theset, ans ass & ress & $5000,61000,000$ & $20 s e s$ & 1.526,0000000 & or & 5. $-11,1480000000$ & .5565 & 516052,0000000 & 15.925 \\
\hline 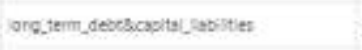 & s96 ent,p00,000 & 30465 & 39:0007,00000000 & 27.125 & 391735000902 & 25015 & 597,207900000 & 29.900 & $56,660,050,000$ & $7 \mathrm{~s}$ & 4-1,928,000,000 & -2065 & 5-3,472,0500005 & -3.575 \\
\hline 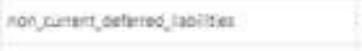 & s. & 0.006 & s- & 20005 & $83221,000,000$ & a.8s & {$[34,345,950,000$} & 2,as & s. & & $5.3223 .000,050$ & toposes & $5,-31,117,000,050$ & .90615 \\
\hline 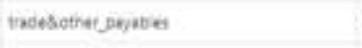 & $521+170000000$ & sives & 528565.0000000 & LTS & 53.389000000 & Q185 & a: & $\cos s$ & 3.+575.800050 & $.5 s$ & $5-404000,000$ & .12045 & $530369,000,000$ & \\
\hline 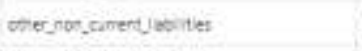 & 526.3280000000 & 0.150 & 120,956,500,000 & 6.196 & S1176s,000,000 & 5054 & E2911,000000 & 2350 & 15.362000,000 & $26 \mathrm{~s}$ & $59,793,060,000$ & 87,718 & 52,254050000 & $2529 \mathrm{~s}$ \\
\hline 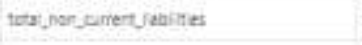 & $5131,157,000,000$ & 47.295 & 5142319,050000 & 42045 & 5141,712000000 & $\operatorname{sem}$ & E) 42558000,000 & I2.25 & $510,847,2020050$ & As & ss9t:s000000 & o.tizk & $5+234,050,000$ & ases: \\
\hline tharesocisisenuly & $565,339,003000$ & 2017 & $592,56,008,050$ & $26 \mathrm{T3s}$ & 1197,447,000,000 & $2930 \mathrm{~s}$ & \$1 $134047,000,000$ & $35 \mathrm{man}$ & 5.-25, 49,000,000, & ats & $5-16,659,000,000$ & .t935s & 5.26,950,000,000 & taoms \\
\hline
\end{tabular}


International Journal of Current Science Research and Review

ISSN: 2581-8341

Volume 04 Issue 07 July 2021

DOI: 10.47191/ijcsrr/V4-i7-21, Impact Factor: 5.825

IJCSRR@ 2021

www.ijesrr.org

Table 10. Microsoft Balance sheet vertical and horizontal analysis (Adapted from the annual reports of Microsoft Corp.)

\begin{tabular}{|c|c|c|c|c|c|c|c|c|c|c|c|c|c|c|}
\hline inecictor os & amourzases & pecemperaxa & movertes: & percorenspeloits & mosintzath & percentspezon & smouratert & Dercentogexis? & ctungetozo & 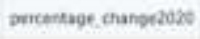 & chanverons & 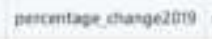 & Dargeante & 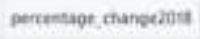 \\
\hline Tots nivets & 500751,800,000 & 1000005 & $5200,58,000,000$ & 1000005: & 1236041003000 & $100505 \%$ & $5241,206,000000$ & $100005:$ & $514,51050,000$ & as & $527,704,0000000$ & 19.706 & $\$ 17,762,000,000$ & $237 \mathrm{~N}$ \\
\hline 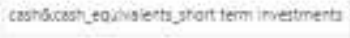 & 1196327000000 & assis & 11790,019,050,000 & $460 \mathrm{~s}$ & SI1217ud000000 & sicos & $5132.90: 000.000$ & $1516 \%$ & 527000000000 & 26 & $551,500,900$ & ADAS & 5787,0000008 & $25 \%$ \\
\hline recovaber & 5120110000000 & 10625 & 529524003050 & $10: 300$ & $522,461,0000000$ & $10.23 \mathrm{~s}$ & 5117อ2000800 & 2.24 & 524670000000 & is & 33041000,000 & 13 AOS & 56,080,000,000 & 232005 \\
\hline mentor & 51,4050000050 & $069 \%$ & 220610000000 & ars & 526620500000 & 1900 & $52 \times 86000000$ & 29806 & $5+6650000005$ & -85 & $3-5960000000$ & $.225 \%$ & 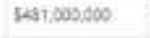 & 22055 \\
\hline athecarent basts & $51+42,000000$ & 3815 & 510,740009050 & 3545 & $5 \pi 51000000$ & 2815 & 44897,000000 & 2095 & 47 338003005 & 135 & $33,305000,000$ & $5029 \mathrm{~s}$ & $51,854,000,050$ & 37.8ES \\
\hline tatesomempeneth & 5182965300,000 & 62975 & $515,552,000,000$ & 6125 & Shentes 003050 & 6555 & 315p:45,, 004090 & 60305 & 56383000000 & 4 & 55.8500000000 & 3,75 & $59,8+1,0020002$ & $E \pm 45$ \\
\hline retpof & 352004900,000 & 17.565 & H3:56600050 & $1330 \%$ & 336 146001000 & ases & 221736000000 & ists. & 59045000005 & $21 \mathrm{~s}$ & 52710,050002 & 2 ziass & $512+12000,000$ & 52309 \\
\hline 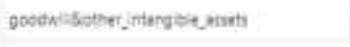 & $150,358050,0000$ & 2675 & 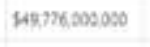 & 17375 & $145,796.0000000$ & 10.905 & $3+52280000000$ & 187275 & 56110000000 & 15 & 3E 040,020000 & 13816 & $5-1,4920000000$ & .3560 \\
\hline 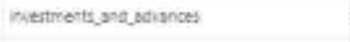 & $52,965003,030$ & deass & $82.649,000,008$ & EBES & st $862,050,002$ & 0,25 & IE, $823,000,080$ & $250 \%$ & 131E0000950 & 128 & 17570000000 & $42 \pi$ & $5,-161050500$ & $\operatorname{tenan}$ \\
\hline othe son covient assets & $513,1310000,000$ & 4365 & $514,723,002,050$ & 5.148 & 57,420800003 & 2.685 & ite.2S0.0020050 & 2396 & f-t,595030,000: & -115 & $57234,000,005$ & 97348 & S1, $192,005,050$ & 12078 \\
\hline 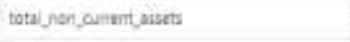 & $51+\$ 396000000$ & 3etsv & st11,004,000,000 & $3674 \mathrm{~s}$ & SaE teteos ovo & $3445 \mathrm{~K}$ & sat 235000000 & 1ajon & 58302000030 & $8 \%$ & 521,8190000000 & $24,4 \mathrm{es}$ & $57.551,000,000$ & 2796 \\
\hline tate janites & 5193027000000 & 62.74 & 5isc 226500,000 & 64285 & $5176 \div 34000050$ & gans & Sterens son 03s & iasts & $5-1,279050,000$ & -75 & sa,0se,000,000 & 4.005 & $57,450,000,050$ & ans \\
\hline periabes & 51a6es:050:000 & 4875 & $515,00,000,000$ & $525 \%$ & 5า2756001000: & tis\% & 58205,200000 & 1005 & 5.s57,000,000 & $-3 \mathrm{~N}$ & $54,309030,000$ & 45136 & $\$ 2,939,000,000$ & 30.875 \\
\hline 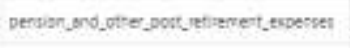 & 57.970000000 & 2615 & Hopo,000,000 & $230 \mathrm{~s}$ & SA 1020000,000 & 2365 & \$s,t19,000,000 & 2475 & 51044000 000 & 154 & 5727,0300000 & $1191 \%$ & 5284005,000 & $490 \mathrm{si}$ \\
\hline cuntendert & $53,749,002,050$ & 1245 & B,518,000,000 & 1905 & $50,998,080,000$ & $1,1,46$ & $370121,000,000$ & 4280 & 4., $, 767,030,000$ & -320 & 31510080,000 & 5,950 & $\$-4,123000,000$ & .60500 \\
\hline cuntricoterenes-jobities & sue, $000,050,000$ & 11,955 & 132876.0020000 & Hase & $\$ 28,958,000,000$ & mes: & $\$ 34102,0005000$ & $1415 \%$ & 53,324002030 & 105 & $53,77,000,000$ & 13095 & 15.5.152,000,000: & . wosts \\
\hline otteccurert/boittes & $512027,050,000$ & 3395 & $19,351,000,008$ & $325 \%$ & 99744000000 & 3.366 & If 2020052050 & 2005 & เ675,030,050 & is & $9857,000,000$ & o.ses & $52,45,000000$ & 39205 \\
\hline tatchermudeites & ม2.370.090.000 & sess & 569.420000 .950 & 24235 & $35 t+38.0000000$ & $2260 \mathrm{~s}$ & 164527552000 & 26.75: & 52,20000000030 & 4 & 50,932000005 & $16 \mathrm{eg}$ & $5-6,039,0500000$ & asen \\
\hline 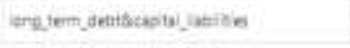 & $162 x \sin 030,000$ & 22.325 & 572459000050 & ses & monstatosess & nowe & cean $0000 \times 00$ & H.358 & 5.5102050000 & -88 & $5-4900,000,000$ & $4.3 n$ & 11, & 2256 \\
\hline nengunertitsethentivaiten & 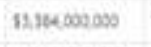 & 1,125 & Hinessososs & 2005 & Hestoso,000 & thes & 610950.050,000 & 4.85: & H., 289050000 & $29 \%$ & $3407,0000.000$ & 9340 & 10,552000200 & enass \\
\hline 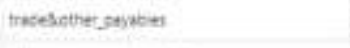 & 529,52500000 & 975 & $229,12000,000$ & 1asss & $32206,001,000$ & nes & i. & 20.98 & 4-130000000 & -15 & $1-653,000,000$ & .2165 & $5802.65 .090,059$ & \\
\hline 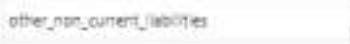 & $570.652900,000$ & ssss: & 17541000000 & 2uss & 55211980,000 & 2014 & f17, $, 184050,009$ & 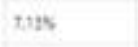 & $55,051,000,000$ & 405 & 2.570,000,005 & ASABS & $5,11,972005000$ & .62 .865 \\
\hline tath_nonavert/jabices & 5170,699000000 & 36745 & m11606, 0000000 & 20005 & 317,060000000 & 4058 & E104,15,0000050 & 43210 & $5.4109,9009000$ & 45 & $1-28360000000$ & 2415 & 511.477 .050 .050 & 120045 \\
\hline 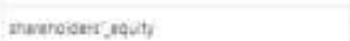 & itis 3ata00,000 & $3926 \mathrm{~s}$ & F502:33:0500000 & $3 \mathrm{~B}, 7 \mathrm{~s}$ & 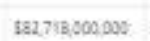 & 3.965 & 1723540000000 & 30055 & 515.974035090 & 165 & $319,8+2000000$ & 23rs & S10.324.000.008 & 16208 \\
\hline
\end{tabular}


International Journal of Current Science Research and Review

ISSN: 2581-8341

Volume 04 Issue 07 July 2021

DOI: 10.47191/ijcsrr/V4-i7-21, Impact Factor: 5.825

IJCSRR @ 2021

www.ijesrr.org

Figure 6. Apple and Microsoft balance sheet visualizations for 2017-2020
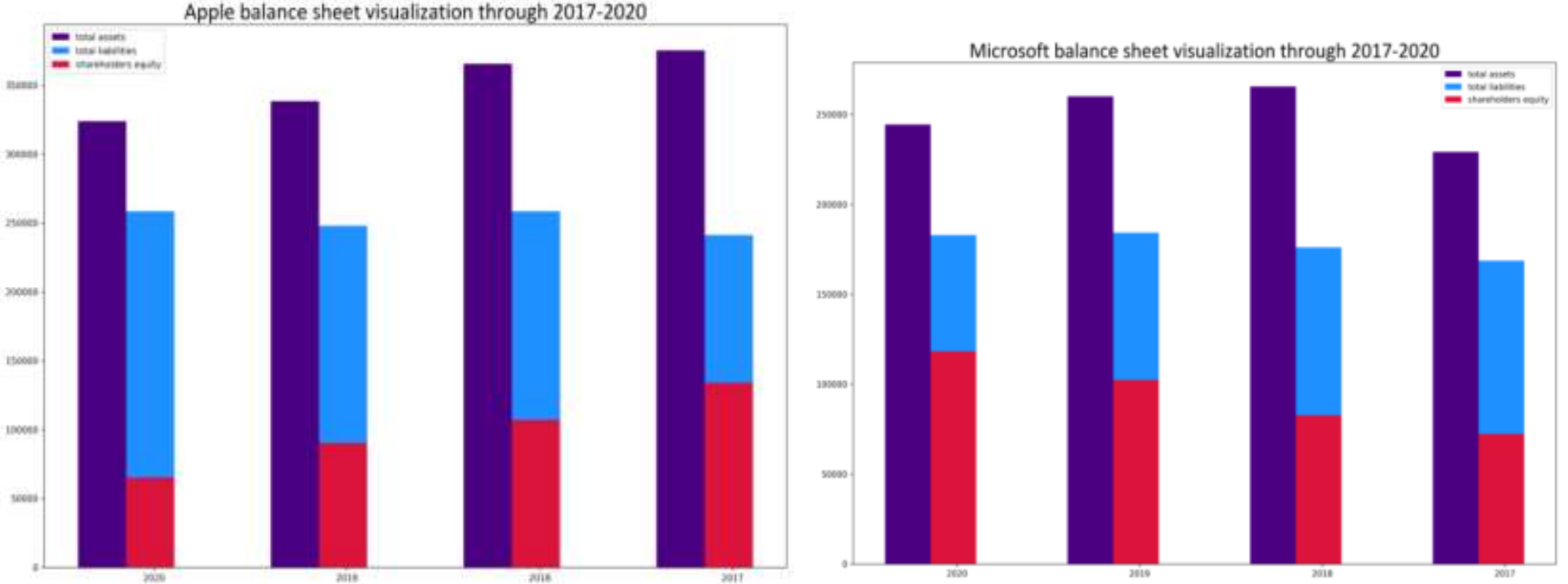
International Journal of Current Science Research and Review

ISSN: 2581-8341

Volume 04 Issue 07 July 2021

DOI: 10.47191/ijesrr/V4-i7-21, Impact Factor: 5.825

IJCSRR @ 2021

www.ijesrr.org

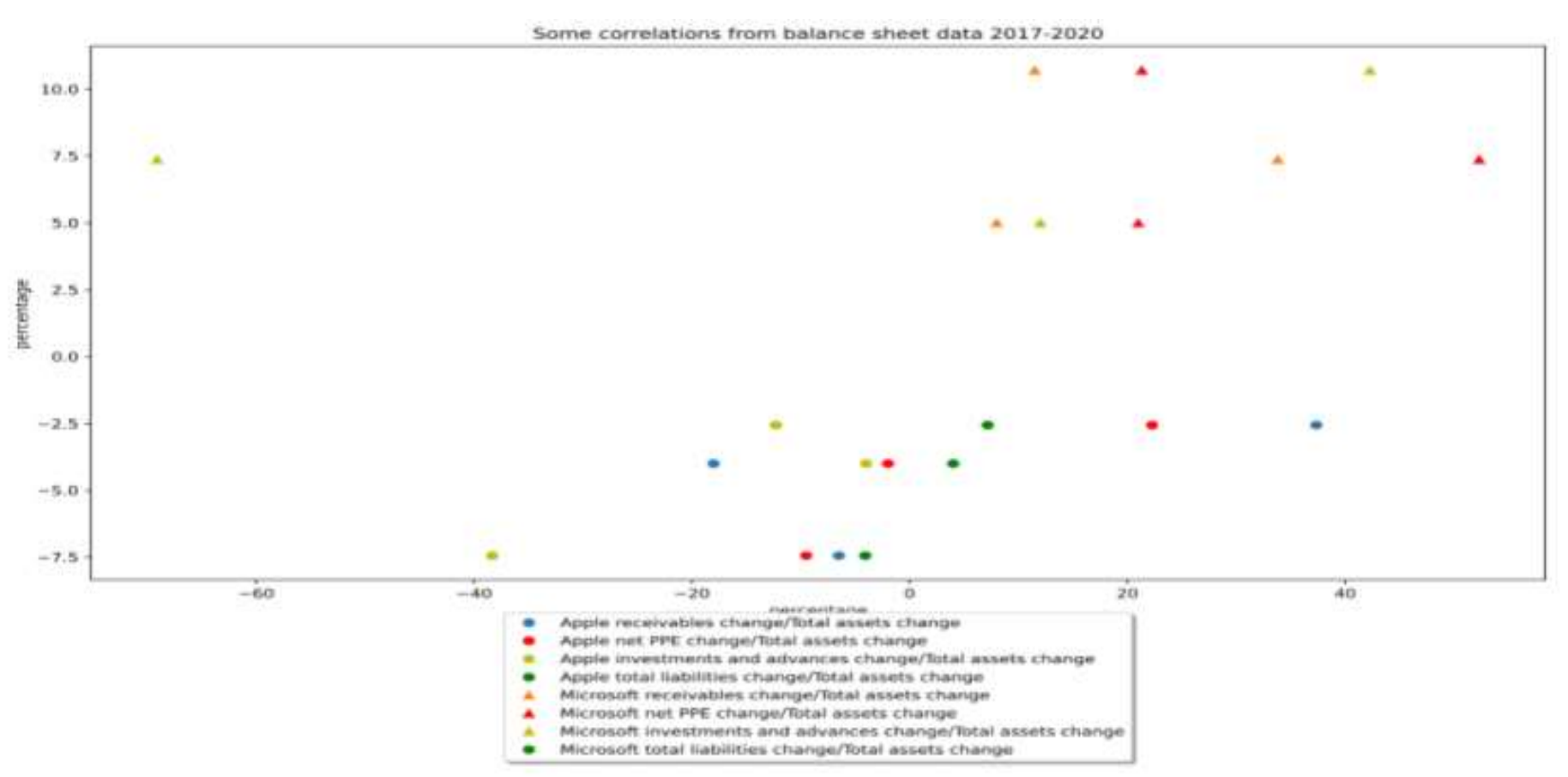

CASH FLOW STATEMENT ANALYSIS FOR 2017-2020

Both Apple and Microsoft has improved their cash flows since 2017, except investing cash flows and financing cash flows, which were decreasing ever since. 
International Journal of Current Science Research and Review

ISSN: 2581-8341

Volume 04 Issue 07 July 2021

DOI: 10.47191/ijcsrr/V4-i7-21, Impact Factor: 5.825

IJCSRR@ 2021

www.ijesrr.org

Table 11. Apple Inc. Cash flow statement vertical and horizontal analysis_(Adapted from the annual reports of Apple)

\begin{tabular}{|c|c|c|c|c|c|c|c|c|c|c|}
\hline Indicator_CFS & amount2020 & amount2019 & amount2018 & amount2017 & change2020 & percentage_change 2020 & change2019 & percentage_change 2019 & change 2018 & percentage_change 2018 \\
\hline cash_and_cash_equivalents_beginning_of_the_year & $\$ 50,224,000,000$ & $\$ 25,913,000,000$ & $\$ 20,289,000,000$ & $\$ 20,484,000,000$ & $\$ 24,311,000,000$ & $93.82 \%$ & $\$ 5,624,000,000$ & $27.72 \%$ & $\$-195,000,000$ & $-0.95 \%$ \\
\hline operating_cash_flow & $\$ 80,674,000,000$ & $\$ 69,391,000,000$ & $\$ 77,434,000,000$ & $\$ 63,598,000,000$ & $\$ 11,283,000,000$ & $16.26 \%$ & $\$-8,043,000,000$ & $-10.39 \%$ & $\$ 13,836,000,000$ & $21.76 \%$ \\
\hline net_income_from_continuing_operations & $\$ 57,411,000,000$ & $\$ 55,256,000,000$ & $\$ 59,531,000,000$ & $\$ 48,351,000,000$ & $\$ 2,155,000,000$ & $3.90 \%$ & $\$-4,275,000,000$ & $-7.18 \%$ & $\$ 11,180,000,000$ & $23.12 \%$ \\
\hline depreciation_amortization_depletion & $\$ 11,056,000,000$ & $\$ 12,547,000,000$ & $\$ 10,903,000,000$ & $\$ 10,157,000,000$ & $\$-1,491,000,000$ & $-11.88 \%$ & $\$ 1,644,000,000$ & $15.08 \%$ & $\$ 746,000,000$ & $7.34 \%$ \\
\hline deferred_tax & $\$-215,000,000$ & $\$-340,000,000$ & $\$-32,590,000,000$ & $\$ 5,966,000,000$ & $\$ 125,000,000$ & $-36.76 \%$ & $\$ 32,250,000,000$ & $-98.96 \%$ & $\$-38,556,000,000$ & $-646.26 \%$ \\
\hline stock_based_compensation & $\$ 6,829,000,000$ & $\$ 6,068,000,000$ & $\$ 5,340,000,000$ & $\$ 4,840,000,000$ & $\$ 761,000,000$ & $12.54 \%$ & $\$ 728,000,000$ & $13.63 \%$ & $\$ 500,000,000$ & $10.33 \%$ \\
\hline other_non_cash_items & $\$-97,000,000$ & $\$-652,000,000$ & $\$-444,000,000$ & $\$-166,000,000$ & $\$ 555,000,000$ & $-85.12 \%$ & $\$-208,000,000$ & $46.85 \%$ & $\$-278,000,000$ & $167.47 \%$ \\
\hline working_capital & $\$ 5,690,000,000$ & $\$-3,488,000,000$ & $\$ 34,694,000,000$ & $\$-5,550,000,000$ & $\$ 9,178,000,000$ & $-263.13 \%$ & $\$-38,182,000,000$ & $-110.05 \%$ & $\$ 40,244,000,000$ & $-725.12 \%$ \\
\hline Investing_cash_flow & $\$-4,289,000,000$ & $\$ 45,896,000,000$ & $\$ 16,066,000,000$ & $\$-46,446,000,000$ & $\$-50,185,000,000$ & $-109.35 \%$ & $\$ 29,830,000,000$ & $185.67 \%$ & $\$ 62,512,000,000$ & $-134.59 \%$ \\
\hline net_PPE_ purchase_and_sale & $\$-7,309,000,000$ & $\$-10,495,000,000$ & $\$-13,313,000,000$ & $\$-12,451,000,000$ & $\$ 3,186,000,000$ & $-30.36 \%$ & $\$ 2,818,000,000$ & $-21.17 \%$ & $\$-862,000,000$ & $6.92 \%$ \\
\hline net_ intangibles_purchase_and_sale & $\$-$ & $\$-$ & $\$-$ & $\$-344,000,000$ & $\$$ - & & \$- & & $\$ 344,000,000$ & $-100.00 \%$ \\
\hline net_business_purchase_and_sale & $\$-1,524,000,000$ & $\$-624,000,000$ & $\$-721,000,000$ & $\$-329,000,000$ & $\$-900,000,000$ & $144.23 \%$ & $\$ 97,000,000$ & $-13.45 \%$ & $\$-392,000,000$ & $119.15 \%$ \\
\hline net_investment_purchase_and_sale & $\$ 5,335,000,000$ & $\$ 58,093,000,000$ & $\$ 30,845,000,000$ & $\$-33,542,000,000$ & $\$-52,758,000,000$ & $-90.82 \%$ & $\$ 27,248,000,000$ & $88.34 \%$ & $\$ 64,387,000,000$ & $-191.96 \%$ \\
\hline net_other_investing_changes & $\$-791,000,000$ & $\$-1,078,000,000$ & $\$-745,000,000$ & $\$ 220,000,000$ & $\$ 287,000,000$ & $-26.62 \%$ & $\$-333,000,000$ & $44.70 \%$ & $\$-965,000,000$ & $-438.64 \%$ \\
\hline Financing_cash_flow & $\$-86,820,000,000$ & $\$-90,976,000,000$ & $\$-87,876,000,000$ & $\$-17,347,000,000$ & $\$ 4,156,000,000$ & $-4.57 \%$ & $\$-3,100,000,000$ & $3.53 \%$ & $\$-70,529,000,000$ & $406.58 \%$ \\
\hline net_issuance_payments_of_debt & $\$ 2,499,000,000$ & $\$-7,819,000,000$ & $\$ 432,000,000$ & $\$ 29,014,000,000$ & $\$ 10,318,000,000$ & $-131.96 \%$ & $\$-8,251,000,000$ & $-1909.95 \%$ & $\$-28,582,000,000$ & $-98.51 \%$ \\
\hline net_common_stock_issuance & $\$-71,478,000,000$ & $\$-66,116,000,000$ & $\$-72,069,000,000$ & $\$-32,345,000,000$ & $\$-5,362,000,000$ & $8.11 \%$ & $\$ 5,953,000,000$ & $-8.26 \%$ & $\$-39,724,000,000$ & $122.81 \%$ \\
\hline Cash_dividends_paid & $\$-14,081,000,000$ & $\$-14,119,000,000$ & $\$-13,712,000,000$ & $\$-12,769,000,000$ & $\$ 38,000,000$ & $-0.27 \%$ & $\$-407,000,000$ & $2.97 \%$ & $\$-943,000,000$ & $7.39 \%$ \\
\hline net_other_financing_charges & $\$-3,760,000,000$ & $\$-2,922,000,000$ & $\$-2,527,000,000$ & $\$-1,247,000,000$ & $\$-838,000,000$ & $28.68 \%$ & $\$-395,000,000$ & $15.63 \%$ & $\$-1,280,000,000$ & $102.65 \%$ \\
\hline changes_in_cash & $\$-10,435,000,000$ & $\$ 24,311,000,000$ & $\$ 5,624,000,000$ & $\$-195,000,000$ & $\$-34,746,000,000$ & $-142.92 \%$ & $\$ 18,687,000,000$ & $332.27 \%$ & $\$ 5,819,000,000$ & $-2984.10 \%$ \\
\hline cash_and_cash_equivalents_end_of_the_year & $\$ 39,789,000,000$ & $\$ 50,224,000,000$ & $\$ 25,913,000,000$ & $\$ 20,289,000,000$ & $\$-10,435,000,000$ & $-20.78 \%$ & $\$ 24,311,000,000$ & $93.82 \%$ & $\$ 5,624,000,000$ & $27.72 \%$ \\
\hline
\end{tabular}


International Journal of Current Science Research and Review

ISSN: 2581-8341

Volume 04 Issue 07 July 2021

DOI: 10.47191/ijcsrr/V4-i7-21, Impact Factor: 5.825

IJCSRR@ 2021

Www.ijcsrr.org

Table 12. Microsoft Cash flow statement vertical and horizontal analysis_(Adapted from the annual reports of Microsoft Corp.)

\begin{tabular}{|c|c|c|c|c|c|c|c|c|c|c|}
\hline Indicator_CFS & amount2020 & amount2019 & amount2018 & amount2017 & change2020 & percentage_change 2020 & change2019 & percentage_change2019 & change2018 & percentage_change 2018 \\
\hline cash_and_cash_equivalents_beginning_of_the_year & $\$ 11,356,000,000$ & $\$ 11,946,000,000$ & $\$ 7,663,000,000$ & $\$ 6,510,000,000$ & $\$-590,000,000$ & $-4.94 \%$ & $\$ 4,283,000,000$ & $55.89 \%$ & $\$ 1,153,000,000$ & $17.71 \%$ \\
\hline operating_cash_flow & $\$ 60,675,000,000$ & $\$ 52,185,000,000$ & $\$ 43,884,000,000$ & $\$ 39,507,000,000$ & $\$ 8,490,000,000$ & $16.27 \%$ & $\$ 8,301,000,000$ & $18.92 \%$ & $\$ 4,377,000,000$ & $11.08 \%$ \\
\hline net_income_from_continuing_operations & $\$ 44,281,000,000$ & $\$ 39,240,000,000$ & $\$ 16,571,000,000$ & $\$ 21,204,000,000$ & $\$ 5,041,000,000$ & $12.85 \%$ & $\$ 22,669,000,000$ & $136.80 \%$ & $\$-4,633,000,000$ & $-21.85 \%$ \\
\hline depreciation_amortization_depletion & $\$ 12,796,000,000$ & $\$ 11,682,000,000$ & $\$ 10,261,000,000$ & $\$ 8,778,000,000$ & $\$ 1,114,000,000$ & $9.54 \%$ & $\$ 1,421,000,000$ & $13.85 \%$ & $\$ 1,483,000,000$ & $16.89 \%$ \\
\hline deferred_tax & $\$-3,620,000,000$ & $\$-3,534,000,000$ & $\$ 13,040,000,000$ & $\$-3,296,000,000$ & $\$-86,000,000$ & $2.43 \%$ & $\$-16,574,000,000$ & $-127.10 \%$ & $\$ 16,336,000,000$ & $-495.63 \%$ \\
\hline stock_based_compensation & $\$ 5,289,000,000$ & $\$ 4,652,000,000$ & $\$ 3,940,000,000$ & $\$ 3,266,000,000$ & $\$ 637,000,000$ & $13.69 \%$ & $\$ 712,000,000$ & $18.07 \%$ & $\$ 674,000,000$ & $20.64 \%$ \\
\hline other_non_cash_items & $\$-219,000,000$ & $\$-792,000,000$ & $\$-2,212,000,000$ & $\$ .59,808,000,000$ & $\$ 573,000,000$ & $-72.35 \%$ & $\$ 1,420,000,000$ & $-64.20 \%$ & $\$ 57,596,000,000$ & $-96.30 \%$ \\
\hline working_capital & $\$ 2,148,000,000$ & $\$ 937,000,000$ & $\$ 2,284,000,000$ & $\$ 69,363,000,000$ & $\$ 1,211,000,000$ & $129.24 \%$ & $\$-1,347,000,000$ & $-58.98 \%$ & $\$-67,079,000,000$ & $-96.71 \%$ \\
\hline Investing_cash_flow & $\$-12,223,000,000$ & $\$-15,773,000,000$ & $\$-6,061,000,000$ & $\$-46,781,000,000$ & $\$ 3,550,000,000$ & $-22.51 \%$ & $\$-9,712,000,000$ & $160.24 \%$ & $\$ 40,720,000,000$ & $-87.04 \%$ \\
\hline net_PPE__purchase_and_sale & $\$-15,441,000,000$ & $\$-13,925,000,000$ & $\$-11,632,000,000$ & $\$-8,129,000,000$ & $\$-1,516,000,000$ & $10.89 \%$ & $\$-2,293,000,000$ & $19.71 \%$ & $\$-3,503,000,000$ & $43.09 \%$ \\
\hline net_ intangibles_purchase_and_sale & $\$$ - & $\$$ & $\$$ & $\$$ & $\$$ - & & $\$$ & & $\$-$ & \\
\hline net_business_purchase_and_sale & $\$-2,521,000,000$ & $\$-2,388,000,000$ & $\$-888,000,000$ & $\$-25,944,000,000$ & $\$-133,000,000$ & $5.57 \%$ & $\$-1,500,000,000$ & $168.92 \%$ & $\$ 25,056,000,000$ & $-96.58 \%$ \\
\hline net_investment_purchase_and_sale & $\$ 6,980,000,000$ & $\$ 540,000,000$ & $\$ 6,557,000,000$ & $\$-12,511,000,000$ & $\$ 6,440,000,000$ & $1192.59 \%$ & $\$-6,017,000,000$ & $-91.76 \%$ & $\$ 19,068,000,000$ & $-152.41 \%$ \\
\hline net_other_investing_changes & $\$-1,241,000,000$ & $\$-$ & $\$-98,000,000$ & $\$-197,000,000$ & $\$-1,241,000,000$ & \#DIV/o! & $\$ 98,000,000$ & $-100.00 \%$ & $\$ 99,000,000$ & $-50.25 \%$ \\
\hline Financing_cash_flow & $\$-46,031,000,000$ & $\$-36,887,000,000$ & $\$-33,590,000,000$ & $\$ 8,408,000,000$ & $\$-9,144,000,000$ & $24.79 \%$ & $\$-3,297,000,000$ & $9.82 \%$ & $\$-41,998,000,000$ & $-499.50 \%$ \\
\hline net_issuance_payments_of_debt & $\$-5,518,000,000$ & $\$-4,000,000,000$ & $\$-10,201,000,000$ & $\$ 31,459,000,000$ & $\$-1,518,000,000$ & $37.95 \%$ & $\$ 6,201,000,000$ & $-60.79 \%$ & $\$-41,660,000,000$ & $-132.43 \%$ \\
\hline net_common_stock_issuance & $\$-21,625,000,000$ & $\$-18,401,000,000$ & $\$-9,719,000,000$ & $\$-11,016,000,000$ & $\$-3,224,000,000$ & $17.52 \%$ & $\$-8,682,000,000$ & $89.33 \%$ & $\$ 1,297,000,000$ & $-11.77 \%$ \\
\hline cash_dividends_paid & $\$-15,137,000,000$ & $\$-13,811,000,000$ & $\$-12,699,000,000$ & $\$-11,845,000,000$ & $\$-1,326,000,000$ & $9.60 \%$ & $\$-1,112,000,000$ & $8.76 \%$ & $\$-854,000,000$ & $7.21 \%$ \\
\hline net_other_financing_charges & $\$-3,751,000,000$ & $\$-675,000,000$ & $\$-971,000,000$ & $\$-190,000,000$ & $\$-3,076,000,000$ & $455.70 \%$ & $\$ 296,000,000$ & $-30.48 \%$ & $\$-781,000,000$ & $411.05 \%$ \\
\hline changes_in_cash & $\$ 2,421,000,000$ & $\$-475,000,000$ & $\$ 4,233,000,000$ & $\$ 1,134,000,000$ & $\$ 2,896,000,000$ & $-609.68 \%$ & $\$-4,708,000,000$ & $-111.22 \%$ & $\$ 3,099,000,000$ & $273.28 \%$ \\
\hline cash_and_cash_equivalents_end_of_the_year & $\$-201,000,000$ & $\$-115,000,000$ & $\$ 50,000,000$ & $\$ 19,000,000$ & $\$-86,000,000$ & $74.78 \%$ & $\$-165,000,000$ & $-330.00 \%$ & $\$ 31,000,000$ & $163.16 \%$ \\
\hline cash and cash equivalents, end of the year & $\$ 13,576,000,000$ & $\$ 11,356,000,000$ & $\$ 11,946,000,000$ & $\$ 7,663,000,000$ & $\$ 2,220,000,000$ & $19.55 \%$ & $\$-590,000,000$ & $-4.94 \%$ & $\$ 4,283,000,000$ & $55.89 \%$ \\
\hline
\end{tabular}

$783^{*}$ Corresponding Author: Prof. Abduraim Vahobov

Volume 04 Issue 07 July 2021

Available at: ijcsrr.org

Page No.-767-786 
International Journal of Current Science Research and Review

ISSN: 2581-8341

Volume 04 Issue 07 July 2021

DOI: 10.47191/ijcsrr/V4-i7-21, Impact Factor: 5.825

IJCSRR@ 2021

\section{www.ijcsrr.org}

Figure 7. Apple and Microsoft cash flow visualizations for 2017-2020
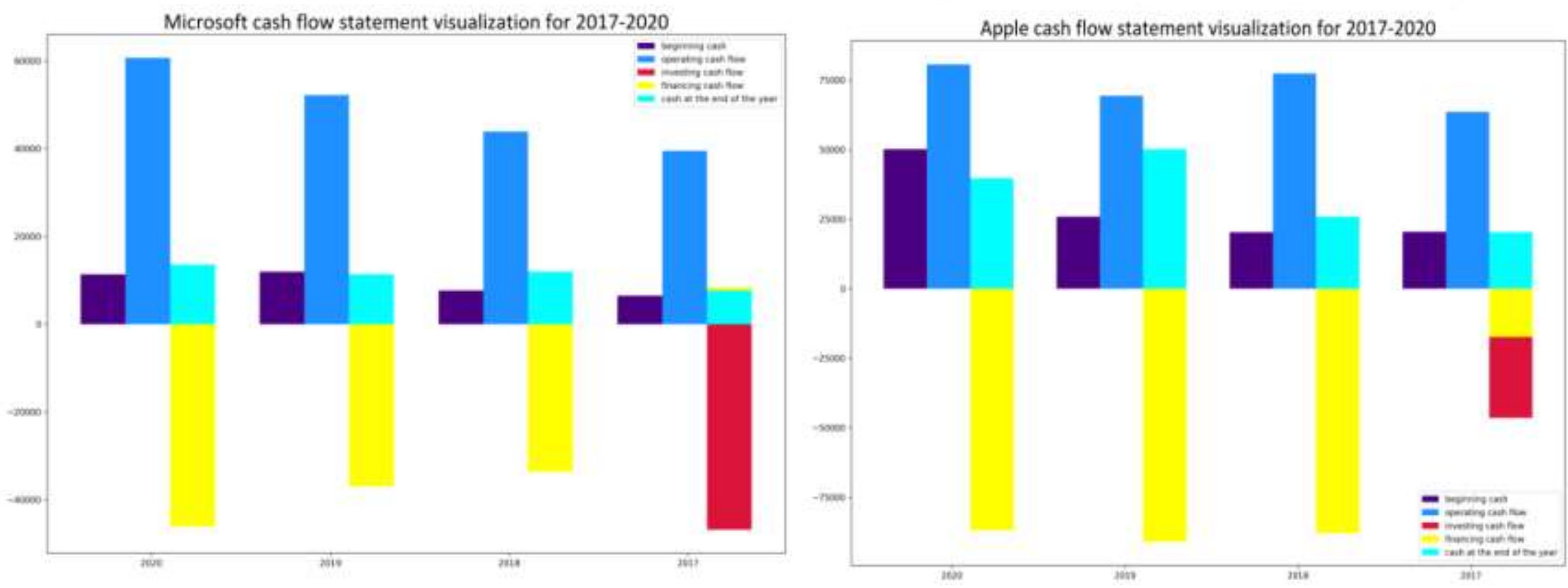
International Journal of Current Science Research and Review

ISSN: 2581-8341

Volume 04 Issue 07 July 2021

DOI: 10.47191/ijcsrr/V4-i7-21, Impact Factor: 5.825

IJCSRR @ 2021

www.ijesrr.org

Table 13. Apple's liquidity, profitability, operating and financial leverage ratios (Adapted from the annual reports of Apple Inc.)

\begin{tabular}{|c|c|c|c|c|}
\hline ratios & 2020 & 2019 & 2018 & 2017 \\
\hline current_ratio & 1.36 & 1.54 & 1.12 & 1.28 \\
\hline quick_ratio & 1.22 & 1.38 & 0.99 & 1.09 \\
\hline gross_profit_margin & 0.38 & 0.38 & 0.38 & 0.38 \\
\hline operating_profit_margin & 0.24 & 0.25 & 0.27 & 0.27 \\
\hline return_on_assets & 0.18 & 0.16 & 0.16 & 0.13 \\
\hline return_on_equity & 0.43 & 0.35 & 0.33 & 0.24 \\
\hline asset_turnover_ratio & 1.26 & 1.12 & 1.07 & 0.84 \\
\hline stock_turnover_period & 8.74 & 9.26 & 8.82 & 12.56 \\
\hline account_receivable_turnover & 7.33 & 5.68 & 5.42 & 6.43 \\
\hline debt to asset ratio & 0.35 & 0.32 & 0.31 & 0.31 \\
\hline
\end{tabular}

Table 14. Microsoft's liquidity, profitability, operating and financial leverage ratios (Adapted from the annual reports of Microsoft Corp.)

\begin{tabular}{|c|c|c|c|c|}
\hline ratios & 2020 & 2019 & 2018 & 2017 \\
\hline current_ratio & 2.52 & 2.53 & 2.9 & 2.48 \\
\hline quick_ratio & 2.33 & 2.35 & 2.74 & 2.37 \\
\hline gross_profit_margin & 0.68 & 0.66 & 0.65 & 0.62 \\
\hline operating_profit_margin & 0.37 & 0.34 & 0.32 & 0.25 \\
\hline return_on_assets & 0.15 & 0.14 & 0.06 & 0.09 \\
\hline return_on_equity & 0.26 & 0.27 & 0.14 & 0.22 \\
\hline asset_turnover_ratio & 0.62 & 0.58 & 0.55 & 0.51 \\
\hline stock_turnover_period & 15.01 & 17.55 & 25.33 & 23.24 \\
\hline account_receivable_turnover & 3.82 & 2.75 & 2.25 & 2.52 \\
\hline debt_to_asset_ratio & 0.24 & 0.27 & 0.32 & 0.36 \\
\hline
\end{tabular}

785 *Corresponding Author: Prof. Abduraim Vahobov

Volume 04 Issue 07 July 2021

Available at: ijcsrr.org

Page No.-767-786 
International Journal of Current Science Research and Review

ISSN: 2581-8341

Volume 04 Issue 07 July 2021

DOI: 10.47191/ijcsrr/V4-i7-21, Impact Factor: 5.825

IJCSRR@ 2021

www.ijcsrr.org

Figure 8. Apple and Microsoft ratio analysis visualizations for 2017-2020
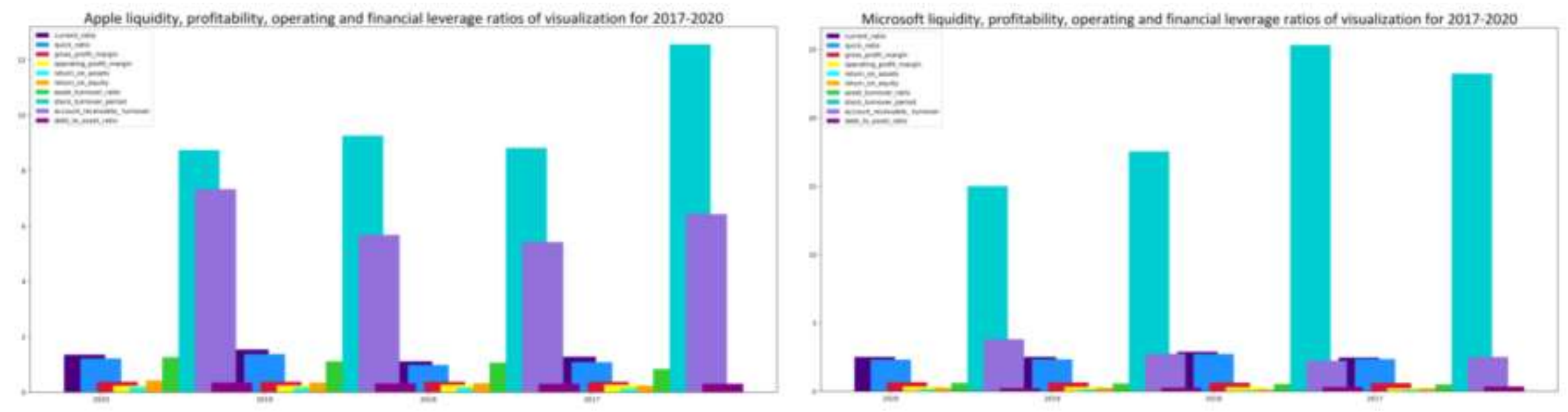

\section{CONCLUSION}

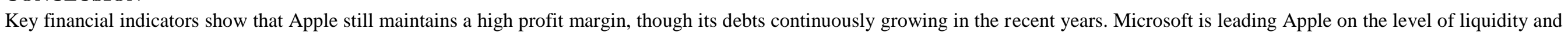
profitability and financial leverage ratios. However, Apple stays unbeatable on its operating ratios.

\section{REFERENCES}

1. https://www.annualreports.com/Company/apple-inc

2. https://www.annualreports.com/Company/microsoft-corporation

3. https://www.macrotrends.net/stocks/charts/

4. https://finance.yahoo.com/

5. https://www.garyfox.co/apple-swot-analysis/

6. https://bstrategyhub.com/swot-analysis-of-microsoft/

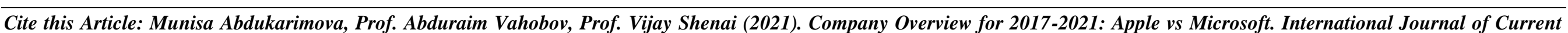
Science Research and Review, 4(7), 767-786 